\title{
FORMULATION OF THE P-EXTENSION FINITE ELEMENTS FOR THE SOLUTION OF NORMAL CONTACT PROBLEMS
}

\author{
I. PÁCZELT ${ }^{a}, \mathrm{~A} \cdot \mathrm{BAKSA}^{a}$ AND T. SzABÓ ${ }^{b}$ \\ ${ }^{a}$ Institute of Applied Mechanics, University of Miskolc, Hungary \\ ${ }^{b}$ Institute of Machine Tools and Mechatronics, University of Miskolc, \\ istvan.paczelt@uni-miskolc.hu attila.baksa@uni-miskolc.hu \\ tamas.szabo@uni-miskolc.hu
}

[Received: August 10, 2020; Accepted: September 8, 2020]

Dedicated to Professor Barna Szabó on the occasion of his eighty-fifth birthday

\begin{abstract}
This work deals with normal contact problems. After a wide literature review, we look for the possibility of achieving a high-precision solution using the principle of minimum potential energy and the Hellinger-Reissner variational principle with penalty and augmented Lagrangian techniques. By positioning of the border of the contact elements, the whole surfaces of the eligible elements fall in contact or in gap regions. This reduces the error of the singularity in the border of the contact domain. Computations with $h-, p$ and $r p$-versions are performed. For the $r p$-version, the pre-fixed number of finite elements are moved so that small elements are placed in one or two element layers at the ends of the contact zone. A number of diagrams and tables showing the convergence of the solution (by increasing the number of polynomial degrees $\mathrm{p}$ ) demonstrate the high efficiency of the proposed solution procedure.
\end{abstract}

Mathematical Subject Classification: 74S05, 74M15

Keywords: contact problems, p-version of finite elements

\section{INTRODUCTION}

The rapid progress in the computer sciences and information technology, the increase of computational speed, and improvements in graphic user-interface software provide new opportunities for modeling physical phenomena with a high degree of reliability and usefulness. It is now possible to perform very large number of arithmetic operations in a short time, making it possible for designers and analysts to employ advanced modeling concepts within the context of an everyday engineering decision-making process.

The solution of contact problems is difficult because the actual contact zone is unknown a priori and must be determined by an iterative procedure which seeks to satisfy not only equilibrium equations and boundary conditions but also one or more inequality contact conditions. Another source of difficulty is that the stress changes very substantially over short distances at the boundaries of the contact zone. An important design objective is to determine the shape of the contacting parts in such a way that the stress singularity will not occur, or the strain energy associated with 
the singularity is within acceptable limits. Therefore the development of efficient and reliable procedures for the numerical treatment of contact is an important and challenging problem.

A number of methods have been published in the field of the mechanical contacts since the appearance of the Hertzian contact theory. Applying the electronic computers, better and better numerical processes were worked out from the late sixties. The paper 1 (Fridman and Chernina, 1967) was an important contribution to the numerical treatment of contact. Their iteration method was based on the search for the minimum of the complementary energy by ensuring positive contact pressure. Its further development could take into regard the relative rigid body displacements of the contacting bodies [2] (Páczelt, 1974). Mathematical programming methods were used to solve the minimum problem in 3] (Conry and Seireg, 1971), 4] (Kalker and von Randen, 1972), [5](Fremond, 1973), [6](Frankavilla and Zienkiewicz, 1975), [7](Haug et al., 1977), 8](Páczelt and Herpai, 1977), 9] (Páczelt, 1979), [10 (Hung and De Saxce, 1980). These methods were applied later as well, see 11] (Klarbring, 1985) and 12](Björkman,1991) for instance.

The generality of the finite element method (FEM) has been utilized efficiently in the solution of contact problems 13 (Chan and Tuba, 1971), 14] (Fredrikson, 1976), 15 (Páczelt, 1976), 16] (Huges et al., 1976), 17] (Bathe and Chaudhary, 1985), 18 (Nour-Omid and Wriggers, 1986), 19 (Chaudhary and Bathe, 1986), 20 (Égert and Altenbach, 1989), 21 (Mottershead et al., 1992), 22 (Papadopoulos and Taylor, 1992).

A Lagrangian multiplier technique was proposed in [15 (Páczelt, 1976) and 16 (Huges et al., 1976), and a penalty method was used first in 23 (Kikuchi et al., 1984). Contact conditions, such as contact and separation, are satisfied accurately for the contacting nodal point pairs in the Lagrangian multiplier method by implying the unknown contact pressure field as a multiplier. The fulfillment of the contact conditions in the solution of the penalty method strongly depends on the value of the penalty parameter. The combination of the two methods was applied in 24] (Simo et al., 1985) for small displacement problems, and in 25] (Ju and Taylor, 1988), 26] (Simo and Laursen, 1992), 27] (Laursen and Simo, 1993) for large displacement cases. The mathematical backgrounds of these methods can be found in 28 (Berteskas, 1989), 29] (Fletcher, 1989).

A good review of static mechanical contact problems is given in [30] (Zhong and Mackerle, 1992).

The error of a finite element solution depends on the element size $(h)$, and on polynomial degree $(p)$ of the approximation.

The majority of the finite element applications use the $h$-version of the FEM. The solution accuracy is improved adaptively in 31 (Nackenhorst, 1995) by the help of the ZZ error estimator, its further development is given by 32] (Wriggers, 1997). Relatively few papers have dealt with the application of the $p$-version method 33 (Szabó and Babuska, 1991) for mechanical contact problems 34] (Lee and Oden, 1993), 35 (Gabbert and Graeff-Winberg, 1994), 36 (Buczkowski et al., 1994), 37] (Páczelt and Szabó, 1995), 38 (Páczelt and Baksa, 2009), 39] (Franke et al., 2010), 
[40 (Konyukhov and Schweizerhof, 2009), 41] (Franke et al., 2011). A quasi-static problem was solved by finite elements with $p=2$ applying mesh refinement in the contact region 34] (Lee and Oden, 1993).

A special $p N h$ type element was used in 35 (Gabbert and Graeff-Winberg, 1994), 36 (Buczkowski et.al., 1994). There is piece-wise linear approximation on the contacting face of the element, and $p$-approximation is applied on the other sides. This sort of approximation provides non smooth stresses inside the element, which is a drawback concerning the efficiency of this element.

The shape optimization of the contacting surfaces was used in 37] (Páczelt and Szabó, 1995) by controlling the contact pressure. The high-quality approximation properties of the $p$-version make it possible to detect the presence of singularity, even weak singularity, by numerical means. This is possible theoretically but not feasible in practice with the $h$-version. The application of the augmented Lagrangian technique was investigated in [42] (Páczelt et al., 1999) concerning the solution accuracy. The contact conditions of the $p$-version elements are checked in the Gauss or Lobatto integration points. As a result of adaptive remeshing the whole sides of the elements are either in the contacting region or in the gap region. This preferable mesh performs no oscillation in the normal stresses along the possible contact region

There are a number of papers dealing with contact problems by the use of variational principles, e.g. [43 (Duvat and Lions, 1972), 44 (Hlavacek et.al., 1980), [45 (Panagiotopoulos, 1985), 46] (Oden and Kikuchi, 1988), 47 (Telega, 1987), 48 (Haslinger and Neittaanmaki, 1988).

Efficient algorithms have been proposed (Nour-Omid and Wriggers, 1986), 49] (Kalker, 1990), and 50] (Raos et al., 1988) in order to solve the system of inequalities established at frictional and frictionless contact problems. A broad range of application possibility of the mathematical programming techniques was analyzed by Klarbring [51]. A review for those papers devoted to the optimization of structures in unilateral mechanical contact is presented in [52] (Hilding et al., 1999).

The $p$-version was employed for locating the boundaries of the contact domain and the boundaries of the sliding-adhesive domain through the detection of the associated singularity. Two different procedures were used. In one case the standard polynomial basis functions were augmented by special basis functions which can approximate singularity 53 (Volpert, 1995), 54 (Volpert et.al., 1997). In the other case the boundaries of the elements were moved, using a special algorithm and a number of error indicators, in such a way that each element in the contact domain would either be in contact, separate, stick or slip along its full length [42] (Páczelt et.al., 1999), 55 (Páczelt and Szabó, 2002). The positioning technique was extended in Baksa et al. 56] for 3D contact problems including friction, where slip, stick zones have been separated. The borders of adhesion and slip zones and also of the outer border of the whole contact domain were approximated by NURBS. In this way the oscillation of the contact pressure and the oscillation of the shear stresses were practically eliminated, resulting in a very accurate solution of the problem. 
In the past decades there have been some valuable books by Laursen 2002 [57, Wriggers 2006 [58, Konyukhov and Schweizerhof 2013 [59], Yastrebov 2013 [60, which describe the numerical treatment of contact problems. In these works not only small displacements and deformations but also large displacements and large deformations are taken into consideration for numerical analysis, both in normal and frictional contact examples.

High order finite elements are used for many different types of problems [61], 62. The research in tribology, analytical solution of contact problems are examined by the book of Goryacheva 63 , and we found a technique to analyze the wear process by $p$-extension finite elements 64 .

In the book 65] many aspects of optimization of contact interaction of rigid, elastic, elastic-plastic bodies are investigated.

There have been some conferences e.g. CMM, Eccomas, HOFEIM, IUTAM, where not only the theoretical questions but also practical problems were analyzed 6669 . Among the $p$-extension finite element computer systems, there is a simulation program called StressCheck developped by ESRD group leaded by Professor B. Szabó [70].

The aim of this investigation is to compare the results of non-frictional contact problems treated by traditional $h$-, $p$ - and with adaptive $h p(r p)$-extension finite elements, and to show the computational algorithm for highly accurate $h p(r p)$-extension.

Using the notations introduced by papers [39], 41], we made some rp-extension computations as well. Here we use the principle of minimum of total potential energy and the mixed Hellinger-Reissner theory, which is based on approximating both the displacements and the stress fields 38 . In this paper a comparison is made to show the advantages of using the Hellinger-Reissner functional. The main question is whether we can get better results with the same number of unknowns of the displacement fields. However, the execution time is much longer because of the eliminating the stress field variables from the evolving stiffness matrix.

In case of the $p$ - and $h p$ - $(r p)$-versions, we use the augmented Lagrangian method for the solution of contact problems. It is assumed that displacements and deformations are small, and can be neglected as regard the adhesion, friction and dynamic effects between the contacting bodies.

This paper is organized as follows. In Section 2 the formulation of the contact problem is presented. In Section 3 two variational methods, minimum of potential energy and Hellinger-Reissner variational principle, are discussed with penalty and augmented Lagrangian form. In Section 4 finite element approximation and the system of algebraic inequalities are given, solving by Kalker-type iteration. Section 5 is dealing with the positioning technique of nodal contact points. Using this technique each eligible contact element along its whole side is either in contact or in the gap regions. Section 6 solves the same boundary value problem of contacting cylindrical bodies with different methods and techniques. These contact problems are solved by $h$-version, $p$-version and $r p$-version techniques. Stress states, distribution of the contact stresses, convergence of the contact radius, resultant of contact force, relative error in energy norm [33] and in contact force are demonstrated in figures. Results of 
the principle of minimum potential energy and the Helliger-Reissner variational principle can be compared in order to choose the better one. In Section 7 Hertz probem with friction will be solved. Finally concluding remarks are provided in Section 8.

\section{Formulation of the CONTACT PROBlem}

Let us consider the contact problem of two elastic bodies $(e=1,2)$. The surfaces of the bodies will be separated into three regions: $S_{u}^{(e)}$ denotes that part of the body where displacements $\boldsymbol{u}_{o}$ are given, in $S_{t}^{(e)}$ the traction $\boldsymbol{t}_{o}$ is applied, while $S_{c}^{(e)}$ represents that part of the bodies where contact is expected. The $S_{c}^{(e)}$ part of the body is called the proposed zone of the contact. The bodies are loaded with the body force $\boldsymbol{b}^{(e)}$, and initial stress $\boldsymbol{T}_{o}^{(e)}$ and initial strain $\boldsymbol{A}_{o}^{(e)}$ too. We are interested in finding the displacement vector field $\boldsymbol{u}$, strain $\boldsymbol{A}$ and stress $\boldsymbol{T}$ tensor fields. In the domain $V^{(e)}$ we have the equilibrium equation

$$
\boldsymbol{T}^{(e)} \cdot \nabla+\boldsymbol{b}^{(e)}=\mathbf{0} \quad \boldsymbol{r} \in V^{(e)},
$$

the strain displacement relationship

$$
\boldsymbol{A}^{(e)}=\frac{1}{2}\left(\boldsymbol{u}^{(e)} \circ \nabla+\nabla \circ \boldsymbol{u}^{(e)}\right) \quad \boldsymbol{r} \in V^{(e)},
$$

and the Hooke's constitutive law

$$
\boldsymbol{T}^{(e)}=\boldsymbol{T}_{o}^{(e)}+\boldsymbol{D}^{(e)} \cdots\left(\boldsymbol{A}^{(e)}-\boldsymbol{A}_{o}^{(e)}\right) \quad \boldsymbol{r} \in V^{(e)},
$$

where $\boldsymbol{D}^{(e)}$ is a fourth order tensor of the material parameters, ". " , " .. ", "o ", are the symbols of a scalar, double scalar and tensor product, respectively, and $\nabla$ is the Hamiltonian differential operator.

The boundary conditions are

$$
\boldsymbol{u}^{(e)}=\boldsymbol{u}_{o} \quad \boldsymbol{r} \in S_{u}^{(e)},
$$

and

$$
\boldsymbol{T}^{(e)} \cdot \boldsymbol{n}^{(e)}=\boldsymbol{t}_{o} \quad \boldsymbol{r} \in S_{t}^{(e)} .
$$

For the examination of the contact/separation conditions in the proposed zone of contact we shall consider the projection of the displacement in a prescribed direction only (e.g., normal to the surface $\boldsymbol{n}_{c}$ ). The contact normal vector $\boldsymbol{n}_{c}$ determines the points $Q_{1}, Q_{2}$ on the corresponding surfaces $S_{c}^{(1)}$ and $S_{c}^{(2)}$, where the two surfaces may contact with each other (see Fig. 1). Therefore the contact surface will be denoted by $S_{c}$.

We denote the displacement projected in the direction $\boldsymbol{n}_{c}$ by $u_{n}^{(e)}=\boldsymbol{u}^{(e)} \cdot \boldsymbol{n}_{c}$, the normal stress by $\sigma_{n}^{(e)}=\boldsymbol{n}^{(e)} \cdot \boldsymbol{T}^{(e)} \cdot \boldsymbol{n}^{(e)}$ and the initial gap between bodies by $h$. We define the distance (gap) after deformation

$$
d=d(\boldsymbol{u})=u_{n}^{(2)}-u_{n}^{(1)}+h \geq 0,
$$

and the contact pressure

$$
p_{n}=-\boldsymbol{n}^{(1)} \cdot \boldsymbol{T}^{(1)} \cdot \boldsymbol{n}_{c}=\boldsymbol{n}^{(2)} \cdot \boldsymbol{T}^{(2)} \cdot \boldsymbol{n}_{c} \cong-\boldsymbol{n}^{(1)} \cdot \boldsymbol{T}^{(1)} \cdot \boldsymbol{n}^{(1)}=-\boldsymbol{n}^{(2)} \cdot \boldsymbol{T}^{(2)} \cdot \boldsymbol{n}^{(2)} \geq 0 .
$$




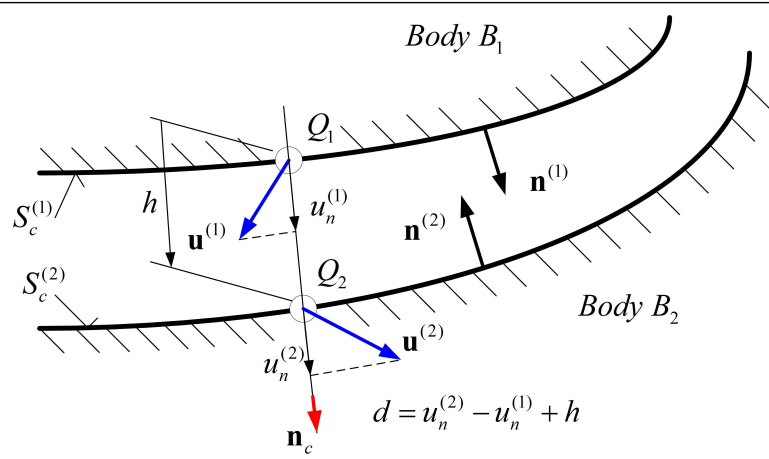

Figure 1. Normal displacements $u_{n}^{(e)}, e=1,2$

Denoting the contact zone by $C$ and the separation (gap) zone by $G\left(S_{c}=C \cup G\right)$, we have

$$
\begin{array}{ccr}
d=0, \quad p_{n} \geq 0 & \boldsymbol{r} \in C, \\
d>0, \quad p_{n}=0 & \boldsymbol{r} \in G, \\
p_{n} d=0 & \boldsymbol{r} \in S_{c} .
\end{array}
$$

From the condition of frictionless contact we have zero tangential stress

$$
\tau^{(e)}=\boldsymbol{e}_{\tau}^{(e)} \cdot \boldsymbol{T}^{(e)} \cdot \boldsymbol{n}^{(e)}=0 \quad \boldsymbol{r} \in S_{c}^{(e)},
$$

where $\boldsymbol{e}_{\tau}^{(e)}$ is a tangential unit vector.

\section{VARIATIONAL FORMULATIONS OF THE CONTACT PROBLEM}

In this section two variation principles will be discussed in parallel.

\subsection{Principles based on the total potential energy and Hellinger-Reissner} functional. For investigation of normal contact problem we can use the principle of minimum potential energy $\Pi(\boldsymbol{u})$ subjected to two types of kinematic conditions: $\boldsymbol{u}=\boldsymbol{u}_{o}$ on $\boldsymbol{r} \in S_{u}$ and $d \geq 0$ on $\boldsymbol{r} \in S_{c}$.

If we use the Hellinger-Reissner functional we have only kinematical constraints for contact conditions: $d \geq 0$ on $\boldsymbol{r} \in S_{c}$

Thus

$$
\min \left\{\Pi(\boldsymbol{u}) \mid \boldsymbol{u}=\boldsymbol{u}_{o}, \quad \boldsymbol{r} \in S_{u}, \quad d \geq 0, \quad \boldsymbol{r} \in S_{c}\right\}
$$

and

$$
\min \left\{\Pi_{R}(\boldsymbol{u}, \boldsymbol{T}) \mid \quad d \geq 0, \quad \boldsymbol{r} \in S_{c}\right\},
$$

which must be solved satisfying the variational inequality $\delta \Pi \geq 0$ or $\delta_{u} \Pi_{R} \geq 0$ and $\delta_{T} \Pi_{R}=0$. The detailed mathematical discussion of this variational inequality and other variational principles can be found in Haslinger and Neittaanmaki [48], Oden and Kikuchi [46] and in Telega 47]. 
Here the total potential energy

$$
\begin{gathered}
\Pi(\boldsymbol{u})=\sum_{e=1}^{2}\left\{\frac{1}{2} \int_{V^{(e)}}\left(\boldsymbol{A}(\boldsymbol{u})-\boldsymbol{A}_{o}\right) \cdots \boldsymbol{D} \cdots\left(\boldsymbol{A}(\boldsymbol{u})-\boldsymbol{A}_{o}\right) \mathrm{d} V+\int_{V^{(e)}} \boldsymbol{A}(\boldsymbol{u}) \cdots \boldsymbol{T}_{o} \mathrm{~d} V-\right. \\
\left.-\int_{V^{(e)}} \boldsymbol{u} \cdot \boldsymbol{b} \mathrm{d} V-\int_{S_{t}^{(e)}} \boldsymbol{u} \cdot \boldsymbol{t}_{o} \mathrm{~d} S .\right\}
\end{gathered}
$$

and the Hellinger-Reissner functional

$$
\begin{gathered}
\Pi_{R}(\boldsymbol{u}, \boldsymbol{T})=\sum_{e=1}^{2}\left\{\int_{V^{(e)}}\left[\boldsymbol{T} \cdots\left(\boldsymbol{A}(\boldsymbol{u})-\boldsymbol{A}_{o}\right)-\frac{1}{2} \boldsymbol{T} \cdot \boldsymbol{C} \cdot \boldsymbol{T}\right] \mathrm{d} V+\right. \\
\left.+\int_{V^{(e)}} \boldsymbol{T} \cdot \boldsymbol{C} \cdot \boldsymbol{T}_{o} \mathrm{~d} V-\int_{V^{(e)}} \boldsymbol{u} \cdot \boldsymbol{b} \mathrm{d} V-\int_{S_{t}^{(e)}} \boldsymbol{u} \cdot \boldsymbol{t}_{o} \mathrm{~d} S \cdot-\int_{S_{u}^{(e)}} \boldsymbol{n} \cdot \boldsymbol{T} \cdot\left(\boldsymbol{u}-\boldsymbol{u}_{o}\right) \mathrm{d} S\right\} .
\end{gathered}
$$

Here $\boldsymbol{C}=\boldsymbol{D}^{-1}$, and ()$^{-1}$ denote the inverse of a tensor. In practice, instead of problem (3.1) and 3.2), functionals (3.3) and (3.4) are extended with penalty functions of the non-penetration contact constraint as

$$
\mathcal{L}^{P E}=\mathcal{L}^{P E}(\boldsymbol{u})=\Pi(\boldsymbol{u})+\frac{1}{2} \int_{S_{c}} c_{n}\left(d^{-}(\boldsymbol{u})\right)^{2} \mathrm{~d} S
$$

and

$$
\mathcal{L}_{R}^{P E}=\mathcal{L}_{R}^{P E}(\boldsymbol{u}, \boldsymbol{T})=\Pi_{R}(\boldsymbol{u}, \boldsymbol{T})+\frac{1}{2} \int_{S_{c}} c_{n}\left(d^{-}(\boldsymbol{u})\right)^{2} \mathrm{~d} S,
$$

where $c_{n}$ is the penalty parameter, and $d^{-}$denotes the negative part of $d$. From the variational equation $\delta_{u} \mathcal{L}^{P E}=0$ and $\delta_{u} \mathcal{L}_{R}^{P E}=0$ we obtain a formula for the contact pressure $p_{n}$.

$$
p_{n}=-c_{n} d^{-}(\boldsymbol{u}) .
$$

The higher the penalty parameter $c_{n}$, the smaller the violation of the non-penetration condition $\lim _{c_{n} \rightarrow \infty} d^{-}(\boldsymbol{u})=0$, i.e., the non-penetration condition $d \geq 0$ is not strictly satisfied.

The correct choice of the penalty parameter is essential, because the condition number of the coefficient matrix increases as the penalty parameter increases. Using $p$-version finite elements [33], [42, $c_{n} \sim 100 E-1000 E$ is recommended, where $\mathrm{E}$ is the Young's modulus.

Combining the Lagrangian and the penalty methods as it was proposed by Simo and Laursen in 26 (Simo and Laursen, 1992), the following augmented Lagrangian 
functionals are obtained

$$
\mathcal{L}^{A U}=\mathcal{L}^{A U}(\boldsymbol{u})=\Pi(\boldsymbol{u})-\int_{C} p_{n} d(\boldsymbol{u}) \mathrm{d} S+\frac{1}{2} \int_{C} c_{n}(d(\boldsymbol{u}))^{2} \mathrm{~d} S,
$$

and

$$
\mathcal{L}_{R}^{A U}=\mathcal{L}_{R}^{A U}(\boldsymbol{u}, \boldsymbol{T})=\Pi_{R}(\boldsymbol{u}, \boldsymbol{T})-\int_{C} p_{n} d(\boldsymbol{u}) \mathrm{d} S+\frac{1}{2} \int_{C} c_{n}(d(\boldsymbol{u}))^{2} \mathrm{~d} S,
$$

where $p_{n}$ is the Lagrangian multiplier, which is kept constant during an iteration loop. From the following two variational equations

$$
\delta_{u} \mathcal{L}^{A U}=0 \text { or } \delta_{u} \mathcal{L}_{R}^{A U}=0
$$

the same formula is obtained for the normal contact stresses

$$
\sigma_{n}^{(1)}\left(\boldsymbol{u}^{1}\right)=\sigma_{n}^{(2)}\left(\boldsymbol{u}^{2}\right)=-\left(p_{n}-c_{n} d(\boldsymbol{u})\right)
$$

and

$$
\sigma_{n}^{(1)}\left(\boldsymbol{T}^{(1)}\right)=\sigma_{n}^{(2)}\left(\boldsymbol{T}^{(2)}\right)=-\left(p_{n}-c_{n} d(\boldsymbol{u})\right) .
$$

During the iteration process, the contact pressure is updated using the formula

$$
p_{n}^{(k)}=\left\langle p_{n}^{(k-1)}-c_{n} d\left(\boldsymbol{u}^{(k)}\right)\right\rangle,
$$

where the operation $\langle\cdot\rangle$ is defined by

$$
\left\langle p_{n}\right\rangle=\frac{1}{2}\left(p_{n}+\left|p_{n}\right|\right) \text {. }
$$

In the $(k+1)$ th iteration loop the contact surface is subjected by $p_{n}^{(k)}$ as an external load in the variational formula:

$$
\delta_{u} \mathcal{L}^{A U}\left(\boldsymbol{u}_{(k+1)}\right)=\delta \Pi\left(\boldsymbol{u}_{(k+1)}\right)-\int_{C^{(k)}} \delta d(\boldsymbol{u})\left(p_{n}^{(k)}-c_{n} d\left(\boldsymbol{u}_{(k+1)}\right)\right) \mathrm{d} S=0
$$

and

$$
\begin{aligned}
\delta_{u} \mathcal{L}_{R}^{A U} & \left(\boldsymbol{u}_{(k+1)}, \boldsymbol{T}_{(k+1)}\left(\boldsymbol{u}_{(k+1)}\right)\right)= \\
& \delta_{u} \Pi_{R}\left(\boldsymbol{u}_{(k+1)}, \boldsymbol{T}_{(k+1)}\left(\boldsymbol{u}_{(k+1)}\right)\right)-\int_{C^{(k)}} \delta d(\boldsymbol{u})\left(p_{n}^{(k)}-c_{n} d\left(\boldsymbol{u}_{(k+1)}\right)\right) \mathrm{d} S=0
\end{aligned}
$$

because of the next variational equation $\delta_{T} \mathcal{L}_{R}^{A U}=0$ we are able to to gain the relation for $\boldsymbol{T}_{(k+1)}=\boldsymbol{T}_{(k+1)}\left(\boldsymbol{u}_{(k+1)}\right)$, i.e.

$$
\boldsymbol{T}_{(k+1)}^{(e)}\left(\boldsymbol{u}_{(k+1)}^{(e)}\right)=\boldsymbol{T}_{o}^{(e)}+\boldsymbol{D}^{(e)} \cdots\left(\boldsymbol{A}^{(e)}\left(\boldsymbol{u}_{(k+1)}^{(e)}\right)-\boldsymbol{A}_{o}^{(e)}\right)
$$

in the approximation process.

REMARK 1: If the second terms of the functionals 3.8 and 3.9 are vanished, we obtain two previous functionals of the penalty methods with $d(\boldsymbol{u})=d^{-}(\boldsymbol{u}) \leq 0$ substitutions. 
- REMARK 2: If the last terms of the functionals $3.8,3.9$ are omitted the funtionals of the Lagrangian multiplier techniques are received back. In these cases $\mathcal{L}^{L A}=\mathcal{L}^{L A}\left(\boldsymbol{u}, p_{n} \geq 0\right), \mathcal{L}_{R}^{L A}=\mathcal{L}_{R}^{L A}\left(\boldsymbol{u}, \boldsymbol{T}, p_{n} \geq 0\right)$ for each functional variational equation or two equations and only one variational inequality are written:

$$
\delta_{u} L^{L A}=0, \quad \delta_{p_{n}} L^{L A} \leq 0 \quad \text { or } \quad \delta_{u} \mathcal{L}_{R}^{L A}=0, \quad \delta_{T} \mathcal{L}_{R}^{L A}=0, \quad-\delta_{p_{n}} \mathcal{L}_{R}^{L A} \geq 0 .
$$

In order to satisfy the contact conditions, positivity of the contact pressure must be ensured.

\section{Finite element formulation}

4.1. Approximated fields. The displacements of the contacting bodies are approximated in the usual form

$$
\boldsymbol{u}^{(e)}=\boldsymbol{u}^{(e)}(\mathbf{x})=\mathbf{N}^{(e)}(\mathbf{x}) \mathbf{q}^{(e)},
$$

where the shape functions $\mathbf{N}^{(e)}(\mathbf{x})$ consist of nodal point modes, side modes and internal modes, and $\mathbf{q}^{e}$ is the vector of displacement parameters, $\mathbf{x}$ is the space coordinates 33. The strain vector is given by the next formula:

$$
\boldsymbol{A}^{(e)}(\boldsymbol{u}) \rightarrow \boldsymbol{\varepsilon}^{(e)}=\varepsilon^{(e)}(\mathbf{x})=\partial \mathbf{u}^{(e)}=\mathbf{B}^{(e)}(\mathbf{x}) \mathbf{q}^{(e)},
$$

where $\mathbf{B}^{e}(\mathbf{x})$ is the strain-displacement matrix. The stress vector can be expressed as

$$
\boldsymbol{T}^{(e)} \rightarrow \boldsymbol{\sigma}^{(e)}=\boldsymbol{\sigma}^{(e)}(\mathbf{x})=\boldsymbol{\sigma}_{o}^{(e)}(\mathbf{x})+\mathbf{D}^{(e)}(\mathbf{x})\left(\mathbf{B}^{(e)}(\mathbf{x}) \mathbf{q}^{(e)}-\varepsilon_{o}^{(e)}(\mathbf{x})\right)
$$

for the minimum principle of total potential energy, where $\mathbf{D}^{(e)}(\mathbf{x})$ is the constitutive matrix, $\varepsilon_{o}^{(e)}(\mathbf{x}), \boldsymbol{\sigma}_{o}^{(e)}(\mathbf{x})$ are the initial strain and stress vectors, respectively. Using (4.1)- (4.3) the total potential energy $(3.3$ can be written in discretized form:

$$
\Pi^{(e)}(\boldsymbol{u}) \rightarrow \Pi^{(e)}\left(\mathbf{q}^{(e)}\right)=\frac{1}{2} \mathbf{q}^{(e), T}\left(\mathbf{K}^{(e)} \mathbf{q}^{(e)}-2 \mathbf{f}^{(e)}\right),
$$

where

$$
\mathbf{K}^{(e)}=\int_{V^{(e)}} \mathbf{B}^{(e), T} \mathbf{D}^{(e)} \mathbf{B}^{(e)} \mathrm{d} V
$$

is the element stiffness matrix, and

$$
\mathbf{f}^{(e)}=\int_{V^{e}} \mathbf{N}^{(e), T} \mathbf{b}^{(e)} \mathrm{d} V+\int_{S_{t}^{(e)}} \mathbf{N}^{(e), T} \mathbf{t}_{o} \mathrm{~d} S+\int_{V^{(e)}} \mathbf{B}^{(e), T}\left(\mathbf{D}^{(e)} \varepsilon_{o}^{(e)}-\boldsymbol{\sigma}_{o}^{(e)}\right) \mathrm{d} V
$$

is the element load vector, $T$ denotes the transpose of a matrix.

In the case of Hellinger-Reissner variational principle the stress is approximated by the next formula

$$
\boldsymbol{T}^{(e)} \rightarrow \boldsymbol{\sigma}^{(e)}=\mathbf{F}^{(e)}(\mathbf{x}) \boldsymbol{\beta}^{(e)},
$$


where $\mathbf{F}^{(e)}(\mathbf{x})$ is an approximation matrix which consists of the same functions that are used for $\mathbf{N}^{(e)}(\mathbf{x})$.

The vector $\boldsymbol{n}^{(e)} \cdot \boldsymbol{T}^{(e)}$ is approximated in the next form

$$
\boldsymbol{n}^{(e)} \cdot \boldsymbol{T}^{(e)} \rightarrow \mathbf{R}^{(e)}(\mathbf{x}) \boldsymbol{\beta}^{(e)} .
$$

The discretized form of Hellinger-Reissner functional (3.4) has the next form:

$$
\begin{aligned}
\Pi_{R}^{(e)}(\boldsymbol{u}, \boldsymbol{T}) & \rightarrow \Pi_{R}^{(e)}\left(\mathbf{q}^{(e)}, \boldsymbol{\beta}^{(e)}\right)= \\
& =\boldsymbol{\beta}^{(e), T} \mathbf{S}^{(e)} \mathbf{q}^{(e)}-\frac{1}{2} \boldsymbol{\beta}^{(e), T} \widetilde{\mathbf{H}}^{(e)} \boldsymbol{\beta}^{(e)}-\mathbf{t}^{(e), T} \mathbf{q}^{(e)}+\boldsymbol{\beta}^{(e), T} \mathbf{g}^{(e)},
\end{aligned}
$$

where

$$
\begin{gathered}
\widetilde{\mathbf{H}}^{(e)}=\int_{V^{(e)}} \mathbf{F}^{T} \mathbf{C} \mathbf{F} \mathrm{d} V, \quad \mathbf{S}^{(e)}=\int_{V^{(e)}} \mathbf{F}^{T} \mathbf{B} \mathrm{d} V-\int_{S_{u}^{(e)}} \mathbf{R}^{T} \mathbf{N} \mathrm{d} S, \\
\mathbf{t}^{(e)}=\int_{V^{(e)}} \mathbf{N}^{T} \mathbf{b} \mathrm{d} V+\int_{S_{t}^{(e)}} \mathbf{N}^{T} \mathbf{t}_{o} \mathrm{~d} S, \\
\mathbf{g}^{(e)}=\int_{S_{u}^{(e)}} \mathbf{R}^{T} \mathbf{u}_{o} \mathrm{~d} S-\int_{V^{(e)}} \mathbf{F}^{T}\left(\boldsymbol{\varepsilon}_{o}-\mathbf{C} \boldsymbol{\sigma}_{o}\right) \mathrm{d} V .
\end{gathered}
$$

From variational equation $\delta_{T} \Pi_{R}^{(e)}=0 \rightarrow \delta_{\beta} \Pi_{R}^{(e)}=0=\delta \boldsymbol{\beta}^{(e), T} \frac{\partial \Pi_{R}^{(e)}}{\partial \boldsymbol{\beta}^{(e)}} \rightarrow \frac{\partial \Pi_{R}^{(e)}}{\partial \boldsymbol{\beta}^{(e)}}=\mathbf{0}$ the stress parameter's vector is

$$
\boldsymbol{\beta}^{(e)}=\left(\widetilde{\mathbf{H}}^{(e)}\right)^{-1} \mathbf{S}^{(e)} \mathbf{q}^{(e)}+\left(\widetilde{\mathbf{H}}^{(e)}\right)^{-1} \mathbf{g}^{(e)}
$$

and the reduced discretized form of $\Pi_{R}^{(e)}$ can be written as follows

$$
\Pi_{R}^{(e)}(\boldsymbol{u}) \rightarrow \Pi_{R}^{(e)}\left(\mathbf{q}^{(e)}\right)=\frac{1}{2} \mathbf{q}^{(e), T}\left(\mathbf{K}^{(e)} \mathbf{q}^{(e)}-2 \mathbf{f}^{(e)}\right),
$$

where the element stiffness matrix

$$
\mathbf{K}^{(e)}=\mathbf{S}^{(e), T}\left(\widetilde{\mathbf{H}}^{(e)}\right)^{-1} \mathbf{S}^{(e)},
$$

and the load vector

$$
\mathbf{f}^{(e), T}=\mathbf{t}^{(e), T}-\mathbf{g}^{(e), T}\left(\widetilde{\mathbf{H}}^{(e)}\right)^{-1} \mathbf{S}^{(e)} .
$$

The gap after deformation is computed by the following projection

$$
d=u_{n}^{(2)}-u_{n}^{(1)}+h=-\mathbf{L}^{(1)}(\mathbf{x}) \mathbf{q}^{(1)}+\mathbf{L}^{(2)}(\mathbf{x}) \mathbf{q}^{(2)}+h=\mathbf{L}(\mathbf{x}) \mathbf{q}+h,
$$

where the matrix of shape functions $\mathbf{L}^{(e)}(\mathbf{x})$ is constructed by the use of $\mathbf{N}^{(e)}(\mathbf{x})$ with $x \in S_{c}$ and the definition of the normal displacement $u_{n}^{(e)}$. The vector of displacement

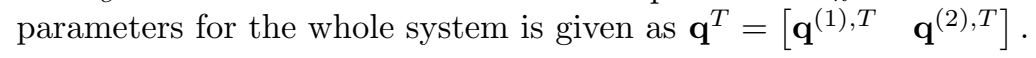


The same penalty terms in $3.8,3.9$ can be written in discretized form

$$
\begin{gathered}
\frac{1}{2} \int_{C} c_{n}(d(\boldsymbol{u}))^{2} \mathrm{~d} S=\frac{1}{2} \mathbf{q}^{T}\left\{\int_{C}\left[\begin{array}{c}
-\mathbf{L}^{(1), T} \\
\mathbf{L}^{(2), T}
\end{array}\right] c_{n}\left[\begin{array}{cc}
-\mathbf{L}^{(1)} & \left.\mathbf{L}^{(2)}\right]
\end{array}\right] \mathrm{d} S \mathbf{q}+\right. \\
\left.+2 \int c_{C}\left[\begin{array}{c}
-\mathbf{L}^{(1), T} \\
\mathbf{L}^{(2), T}
\end{array}\right] h \mathrm{~d} S\right\}+ \text { const }=\frac{1}{2} \mathbf{q}^{T}\left[\begin{array}{cc}
\widetilde{\mathbf{C}}^{11} & -\widetilde{\mathbf{C}}^{12} \\
-\widetilde{\mathbf{C}}^{21} & \widetilde{\mathbf{C}}^{22}
\end{array}\right] \mathbf{q}+ \\
+\mathbf{q}^{T}\left[\begin{array}{c}
-\mathbf{f}_{h}^{(1)} \\
\mathbf{f}_{h}^{(2)}
\end{array}\right]+\text { const }=\frac{1}{2} \mathbf{q}^{T} \widetilde{\mathbf{C}} \mathbf{q}+\mathbf{q}^{T} \mathbf{f}_{h}+\text { const },
\end{gathered}
$$

where $\widetilde{\mathbf{C}}$ is the contact stiffness matrix.

From the same Lagrangian terms in $3.8,3.9$ the load vector $\mathbf{f}_{p}^{e}$ associated to the contact pressure is

$$
\int_{C} d(\mathbf{u}) p_{n} \mathrm{~d} S \rightarrow \mathbf{q}^{T} \int_{C}\left[\begin{array}{c}
-\mathbf{L}^{(1), T} \\
\mathbf{L}^{(2), T}
\end{array}\right] p_{n} \mathrm{~d} S=\mathbf{q}^{T}\left[\begin{array}{c}
-\mathbf{f}_{p}^{(1)} \\
\mathbf{f}_{p}^{(2)}
\end{array}\right]=\mathbf{q}^{T} \mathbf{f}_{p} .
$$

4.2. Discretized functionals. Finally, the discretized funtionals of $\mathcal{L}^{A U}$ and $\mathcal{L}_{R}^{A U}$ expressed with nodal displacements are written in the same form as

$$
\mathcal{L}^{A U}=\mathcal{L}^{A U}(\mathbf{q})=\sum_{e}\left\{\frac{1}{2} \mathbf{q}^{(e), T} \mathbf{K}^{(e)} \mathbf{q}^{(e)}-\mathbf{q}^{(e), T} \mathbf{f}^{(e)}\right\}+\frac{1}{2} \mathbf{q}^{T} \widetilde{\mathbf{C}} \mathbf{q}+\mathbf{q}^{T}\left(\mathbf{f}_{h}-\mathbf{f}_{p}\right)
$$

The algebraic system of equation associated with 4.18 has the following form

$$
\left[\begin{array}{cc}
\mathbf{K}^{1}+\widetilde{\mathbf{C}}^{11} & -\widetilde{\mathbf{C}}^{12} \\
-\widetilde{\mathbf{C}}^{21} & \mathbf{K}^{2}+\widetilde{\mathbf{C}}^{22}
\end{array}\right]^{(k)}\left[\begin{array}{l}
\mathbf{q}^{(1)} \\
\mathbf{q}^{(2)}
\end{array}\right]_{(k+1)}=\left[\begin{array}{c}
\mathbf{f}^{(1)}+\mathbf{f}_{h}^{(1),(k)}-\mathbf{f}_{p}^{(1),(k)} \\
\mathbf{f}^{(2)}-\mathbf{f}_{h}^{(2),(k)}+\mathbf{f}_{p}^{(2),(k)}
\end{array}\right],
$$

in which the matrix $\widetilde{\mathbf{C}}^{i j}$ is modified to fulfill the contact/separation conditions.

The iterational KALKER procedure [49], 37] with the control of the sign of $p_{n}$ can be applied for solving 4.19). The contact conditions are checked in the Gauss or Lobatto integration points of the contact elements during the solution of $(4.19)$. With the updated contact pressure $p_{n}^{(k+1)}$ the integrals 4.16 and 4.17) can be computed repeatedly, that is we have a new penalty (contact) matrix $\mathbf{C}$ and new vectors $\mathbf{f}_{h}$ and $\mathbf{f}_{p}$. The $(k+1)$ th displacements are obtained from the solution of $(4.19)$. The procedure is terminated when the following condition is fulfilled:

$$
\frac{\int_{S_{c}}\left|p_{n}^{(k+1)}-p_{n}^{(k)}\right| \mathrm{d} S}{\int_{S_{c}} p_{n}^{(k+1)} \mathrm{d} S} \leq 10^{-4} .
$$

Remarks:

1. Since $d(\boldsymbol{u})$ is computed in the local coordinate system, the elements which have boundaries on the contact surface, must be transformed from global coordinate system to the local one. The transformation is performed by least squares 
fitting. A detailed explanation of this problem and the numerical reasoning of the transformation can be found in 42 .

2. When the $p$-version is used then accuracy is typically high enough for singularities to induce oscillations in the numerical solutions. The oscillations are minimized when nodes are located at the boundary of the contact zone. The idea of the nodal positioning technique was first published in [42].

3. The system of inequalities according to Lagrangian technique is a Linear Complementary Problem, which can be solved by different algorithms as given in e.g. 11], 21] and 49.

4. In the work 18] a two-level algorithm is employed for solution of the contact problem using Lagrangian multipliers.

\section{Positioning the NOdAl POInts}

One of the advantages of the $p$-version is that for smooth problems only coarse meshes are needed, since the error in energy norm decreases exponentially when the polynomial degree of elements is increased 33] (Szabó and Babuska, 1991). At the border of the contact zone we have a singularity in the stress state. When the ends of contact zone $C$ are not situated in nodal points (in 2D case) then the derivatives of the shape functions cannot have the appropriate jumps there, that is, the singularities induce oscillations in the pressure distribution. By positioning (moving) the nodal points to the ends of contact zone $C$, the jump in the derivatives can be represented in the discretized problem.

The positioning algorithm had been developed for contact problems of a twodimensional problem 42] (Páczelt et.al., 1999). The positioning of the nodal points is performed in one or two phases, depending on the predefined tolerance. The first phase is a rough positioning of contact point to ensure that contact exists in each integration point of the contacting elements. In the second phase the border points are moved based on error indicators in order to increase the accuracy.

5.1. The rough positioning technique. We supposed that the contact zone of two axsymmetric bodies is situated on the left side of the possible contact region. Let $r_{i}, r_{j}$ and $r_{k}$ denote the radial coordinates of the nodal points of the contact element in the original mesh (see Figure 3). We search for the contact (that is $p_{n}>0$ ) from the right-hand direction. The contact elements are integrated in $N$ Gauss or Lobatto points. The star and triangular markers denote, respectively, the $N^{\text {th }}$ integration point and the first contacting integration point from the right side, which is denoted by $I C$. The gap zone is denoted by a single line, the parallel double lines denote the compressed Winkler spring contact zone. The $(\widetilde{r})$ denotes the modified radial coordinate. The natural coordinate of the contact element is denoted by $\xi$.

Checking the contact conditions in integration points is started from the right-hand side. The first contact point $r_{I C}$ is detected in the positive range of the standard coordinate. The location of the new nodal point is found by linear mapping (Step 3).

The algorithm has the following steps:

Step 1. The contact problem is solved with the given mesh. 


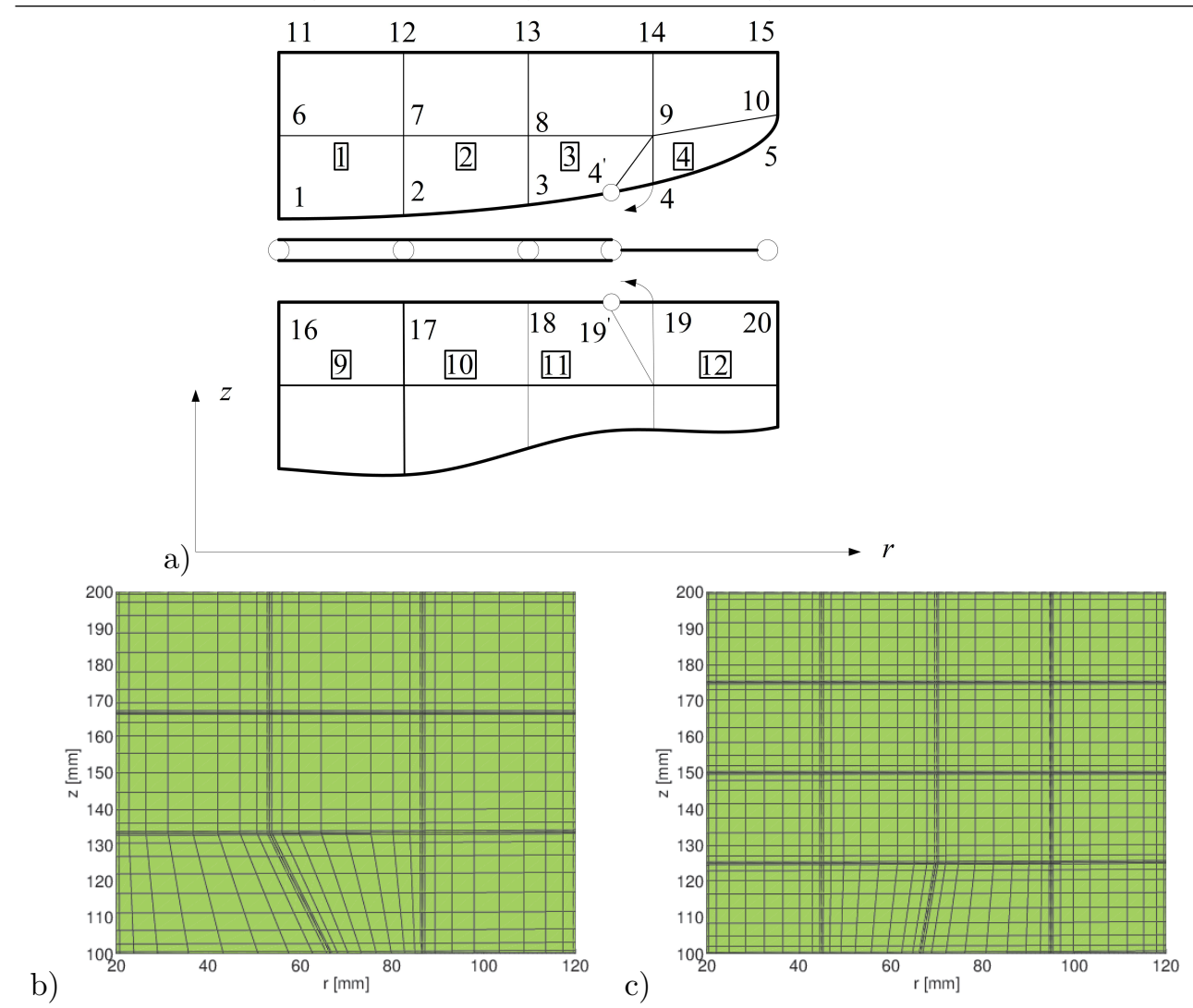

Figure 2. a) nodal points 4 and 19 are moved to $4^{\prime}$ and $19^{\prime}$, elements $1-3$ and $9-11$ are in contact, and between elements 4 and 12 there is no contact; b) modified $(3 \times 3)$ mesh c) modified $(4 \times 4)$ mesh.

Step 2. Search to find the end points of contact region. We seek the first contacting integration point located in $r_{I C}$, where the contact pressure $p_{n}$ is positive.

Step 3 The position of the new end point is calculated by linear mapping based on the position $r_{N}$ of the new $N^{t h}$ integration point:

$$
\widetilde{r}_{j}=\frac{\left[2 \widetilde{r}_{N}-r_{j}\left(1-\xi_{N}\right)\right]}{\left(1+\xi_{N}\right)}
$$

where $\widetilde{r}_{N}$ is computed by the use of the bisection method:

$$
\widetilde{r}_{N}=\left\{\begin{array}{lll}
\frac{1}{2}\left(r_{I C}+r_{N}\right) & \text { if } & \xi_{I C} \geq 0 \\
\frac{1}{2}\left(r_{I C}+r_{\circ}\right) & \text { if } & \xi_{I C}<0
\end{array}\right.
$$

The iteration Steps 1-3 are repeated unless each integration point of the contacting elements is in contact. As a result of this iteration process we have a new mesh, which will be called "the first correct mesh". 


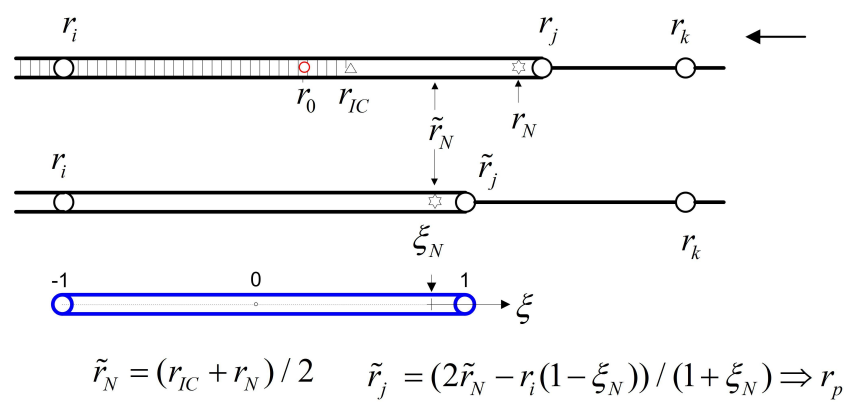

Figure 3. Schematic representation of the right hand side of the contact zone $C$. Calculation of the new position of the point $j$.

5.2. The fine positioning technique. After finding the first "correct mesh" we can further adjust the border of the contact zone $C$. The following indicators are introduced to monitor the fine adjustment of the nodal points:

- contact pressure at the contact border point (zero value is sought)

$$
I_{1}=\min \left|c_{n} \cdot d\left(r_{j}\right)\right|
$$

where $r_{j}$ is the coordinate of the computed contact border point,

- minimum of the potential energy

$$
I_{2}=\min \Pi_{P}
$$

- minimum of the differences between contact pressure and normal stresses

$$
I_{3}=\min \left[\int\left\{\left(\sigma_{n}^{(1)}+p_{n}\right)^{2}+\left(\sigma_{n}^{(2)}+p_{n}\right)^{2}\right\} \mathrm{d} S\right]^{0.5}
$$

- minimum of shear stress on $S_{c}$

$$
I_{4}=\min \left[\int\left\{\left(\tau_{r z}^{(1)}\right)^{2}+\left(\tau_{r z}^{(2)}\right)^{2}\right\} \mathrm{d} S\right]^{0.5},
$$

where the upper and lower bodies are denoted by superscripts 1 and 2, respectively.

In order to minimize the indicators the nodal point is positioned at the vicinity of the border of contact zone determined by the rough positioning technique. That is, the nodal point is moved either to the direction of the $N^{t h}$ integration point of the contacting element, or to the direction of the $1^{\text {st }}$ integration point of the gap element. The minimum of the indicator is searched within the interval determined by these two integration points.

5.3. Position technique assuming friction. The solution of the frictional contact problem is demonstrated in the middle of Figure 4. The end of the contact region is situated between elements $4_{e}$ and $8_{e}$. The border of the stick-slip zones is between elements $2_{e}$ and $6_{e}$. In the point $P_{p}$ the normal stress $\sigma_{z}$ is continuous but the derivatives on the right and left sides are not equal $\left(\frac{d \sigma_{z}}{d r}\right)^{-} \neq\left(\frac{d \sigma_{z}}{d r}\right)^{+}$. 

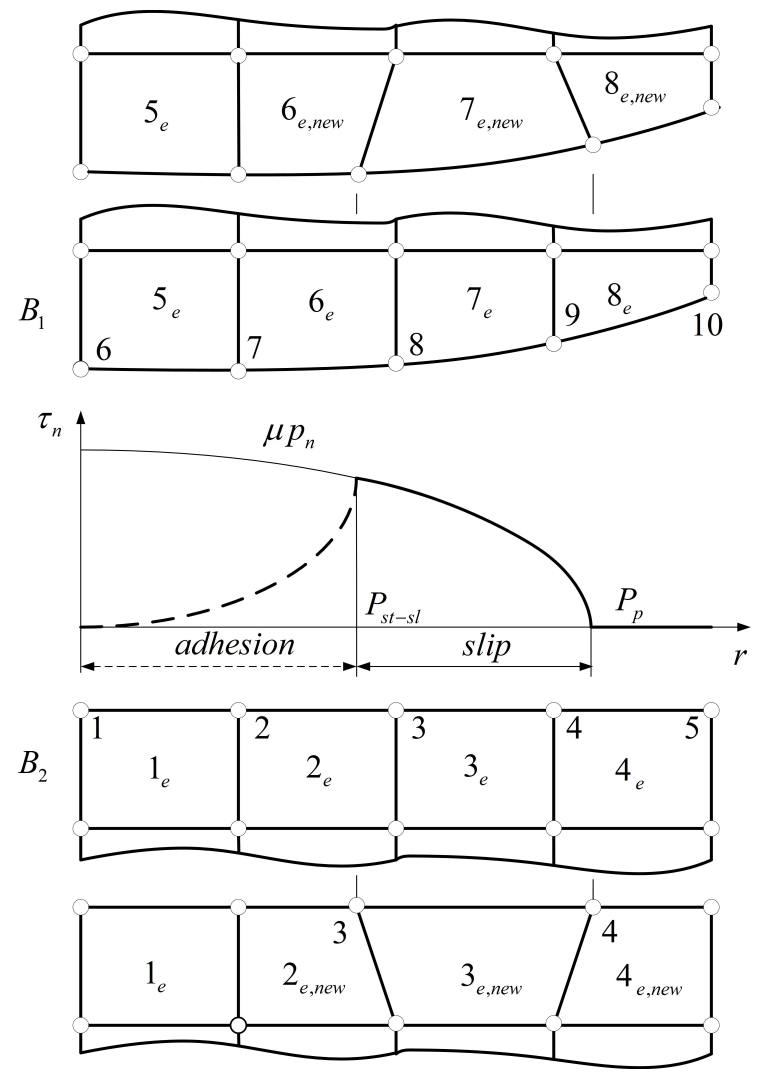

Figure 4. Modification of the mesh at friction contact problem

Since the approximated function is continuous along element edge and the derivatives are also continuous up to order $p-1$ the discrepancy between the derivatives is not represented, which induces oscillations in the results. Therefore new positions for the nodal points 4 and 9 shoul be found. A similar situation takes place for shear stresses in the point $P_{s t-s l}$, which also requires mesh modifications. That is, nodal points 3 and 8 must be moved to left direction. After these positioning steps the elements $2_{e}, 3_{e}, 4_{e}$ and $6_{e}, 7_{e}, 8_{e}$ have new shapes $2_{e, n e w}, 3_{e, n e w}, 4_{e, n e w}$ and $6_{e, \text { new }}, 7_{e, \text { new }}, 8_{e, \text { new }}$, as shown in Figure 4 .

Steps of the algorithm developed for friction contact problems are published in 56 . The shear stresses in the contact zone must satisfy the condition given for the slip surface $\Phi=\left|\tau_{r z}\right|-\mu p_{n} \leq 0$, which is performed with a predictor-corrector iterative algorithm. In the course of the time integration process the predicted value of the shear stress which is proportional to $c_{\tau} \Delta u_{\tau}$ must be calculated, where displacement in tangential direction is given as: $\Delta u_{\tau}=u_{\tau}^{t+\Delta t}-u_{\tau}^{t}$, at time $t$, with time step $\Delta t$. The number of integration points along an element side is denoted by Nlobatto. For the example let us suppose that on the left side of the element there is a stick zone, i.e., 
for integration Lobatto points (ilobatto $=1, \ldots$, istick) the condition of slip surface is satisfied: $\Phi^{\text {pred }}($ ilobatto $) \leq 0$. However, for the rest of the integration points the condition of the slip surface is not satisfied: $\Phi^{\text {pred }}($ ilobatto $)>0$, (ilobatto $=$ istick $+1, \ldots$, Nlobatto $)$. A return mapping algorithm is applied to satisfy the slip surface condition. This means that the right node point of the element must be moved to the left direction. At the end of iteration in each integration point of element $2_{e, n e w}$ there is stick: (ilobatto $=1, \ldots$, Nlobatto), i.e., the whole element side is in stick state.

\section{EXAMPLES}

Let us consider two contacting cylindrical bodies with equal dimensions (inner diameter $D_{i}=2 r_{i}=40 \mathrm{~mm}$ and outer diameter $D_{u}=2 r_{u}=240 \mathrm{~mm}$ and height $h_{c y l}=100 \mathrm{~mm}$, see Fig. 5 . The meridian curve of the contacting surface of the upper body is given by the following function

$$
h=0.0004(r-20)^{2}
$$

which is practically the function of the initial gap between the contacting bodies. There is no friction between the contacting bodies. The displacement is prescribed

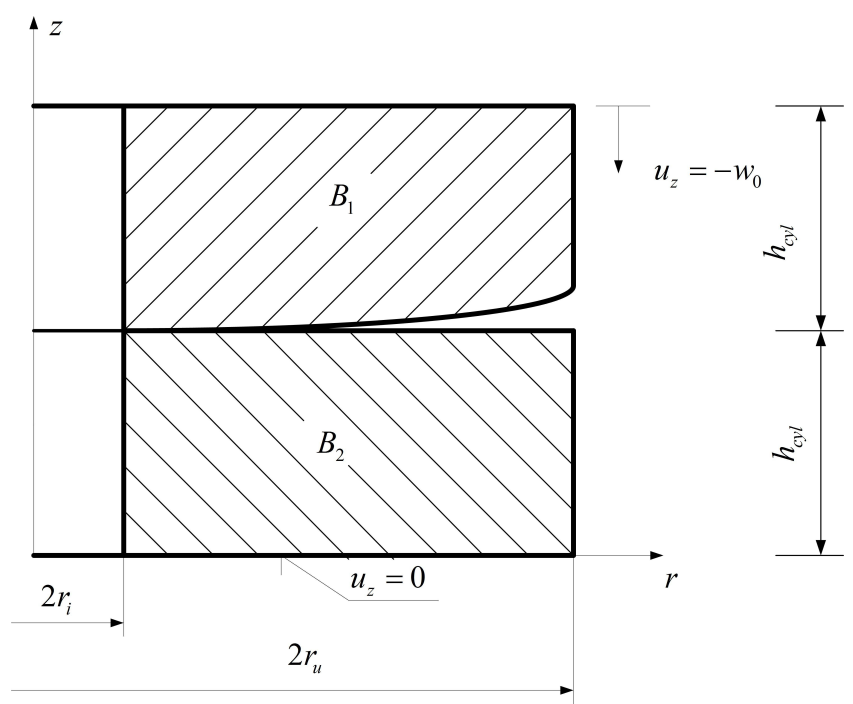

Figure 5. Two cylinders in contact. Initial gap $h=0.0004(r-20)^{2}$

on the top of the upper body in vertical direction $w_{0}=0.15 \mathrm{~mm}$. Elastic material properties are given by Young's modulus $E=200000 \mathrm{MPa}$ and Poisson's ratio $\nu=$ 0.3 . The boundary of the contact zone for body $B_{1}$ is defined by the blending function method 33 .

The contact problem was solved by $h$-version, $p$-version and $h p(r p)$-version techniques using the error indicator $I_{1}$ for finding the radial coordinate of the contact border $r_{p}$. 
The contact problems are solved as detailed below. The positioning technique introduced the previous section is used in all of the examples. The contact conditions are checked in the Gauss integration points. The penalty parameter is chosen as $c_{n}=1000 E$. The analyzed tasks are identified by the type of the applied mesh (number of elements in columns $\times$ rows).

Firstly, the problem is discretized with classical quadratic (low polynomial order) elements, i.e. $h$-version finite element method is used.

6.1. Task 1. For solving the contact problem the Hellinger-Reissner variational principle is applied using penalty technique. For the sake of convenience, instead of using the name Hellinger-Reissner variational hereafter. The polynomial order $p=2$ is fixed, and the problem is solved by different meshes $4 \times 4,8 \times 8,16 \times 16$ and $32 \times 32$.

Figure 6a shows the final status of the mesh $8 \times 8$, the mesh also contains parametric lines crossing on the Gauss integration points. The distribution of the normal stress $\sigma_{z}$ for body $B_{1}$ is given in Figure $3 \mathrm{~b}$. Figure 6 c shows the results obtained for meshes $8 \times 8,16 \times 16$ and $32 \times 32$. Due to mesh refinement, the contact pressure $p_{n}$ becomes smooth together with normal stress $\sigma_{z}$, but in the separation domain $r \geq r_{p}$ defined by the $p_{n}$, significant normal stresses are present, though in principle it should be zero. For mesh $8 \times 8$, the absolute value of the $\sigma_{z}$ is higher than $10 \mathrm{MPa}$, while for the mesh $32 \times 32$ it does not fall below $7 \mathrm{MPa}$. These stress peaks occur at the end of the contact domain in oscillating form. Such oscillations can be experienced for each subsequent solution, only its extent will vary significantly. The smallest oscillation amplitude is obtained by the $r p$-version technique. It can also be observed that the differences in the contact stresses between the upper and lower bodies are very small.

Convergence diagrams of strain energy and contact border radius $r_{p}$ calculated for polynomial degree $p=2$ and $p=3$ are shown in Figure 7. The change in strain energy is well converged (see Figure $7 \mathrm{a}$ ) but the convergence of contact border radius is a bit slower. In these figures, we refer to converged results with Mesh $7 \times 5$ Type: Imesh $=3$, which have been achieved with far fewer unknowns. The number of unknowns is 12674 for mesh $32 \times 32$ with $p=2$, and it is 4474 for mesh $7 \times 5$ (Type: Imesh $=3$ with $p=8$ ). It is well known 33 that the speed of convergence for $h$-version is twice as slow as for $p$-version, however, the rate of convergence is exponential for $h p(r p)$-version computations.

6.2. Task 2. Here, we investigate the contact solution as a function of polynomial degree $p$. Our aim is to determine the position of the contact border as accurate as possible, i.e. the convergence of the solutions is examined.

The border points of the contact zone are determined by the nodal positioning technique detailed in Section 5 using error indicator $I_{1}$.

Augmented Lagrangian method is applied for solving these contact problems.

In this case computations are performed by $p$-extension finite elements. Here, the meshes of the contacting bodies are fixed, but the polynomial order $p$ is increased from 2 to 8 . The solution can be obtained with the positioning technique introduced in the previous section. We use rectangular elements with truncated space 33 . 
a)

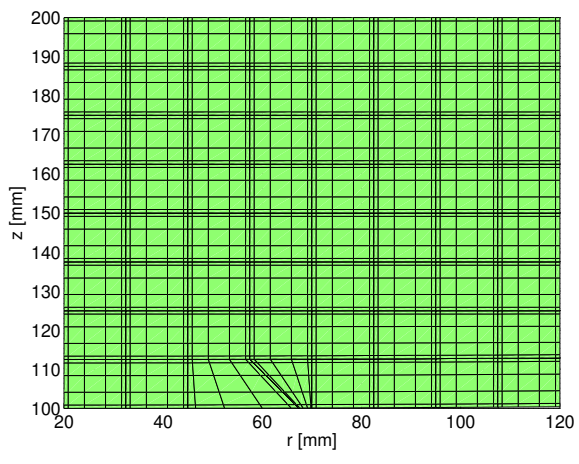

b)
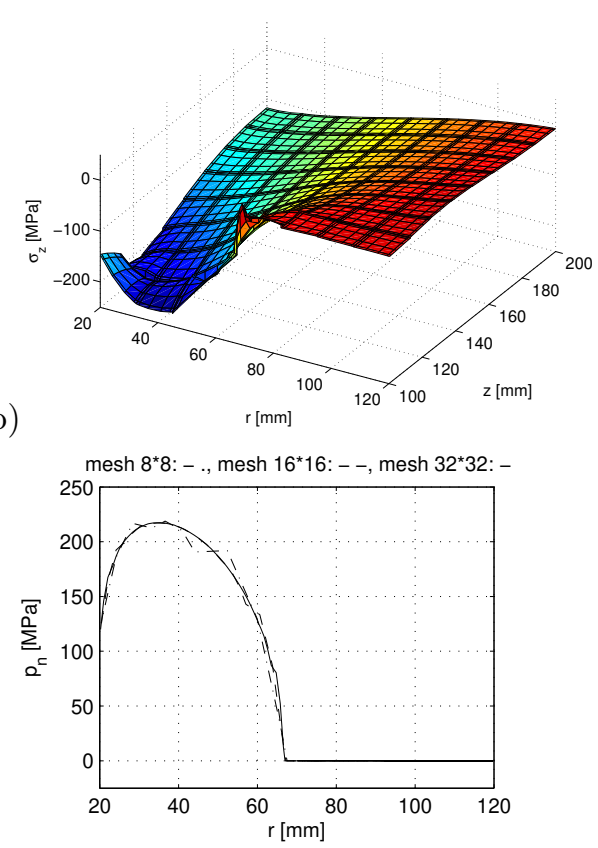

mesh $8 * 8:-$, mesh $16^{\star} 16:--$, mesh $32^{\star} 32$ :

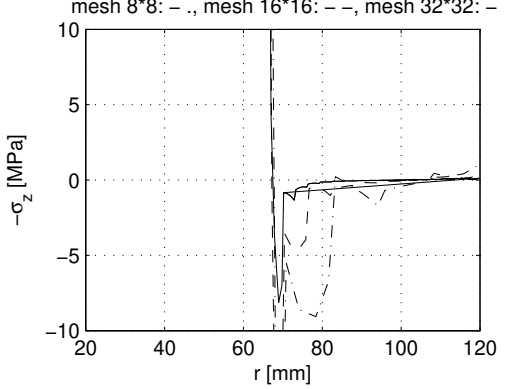

c)
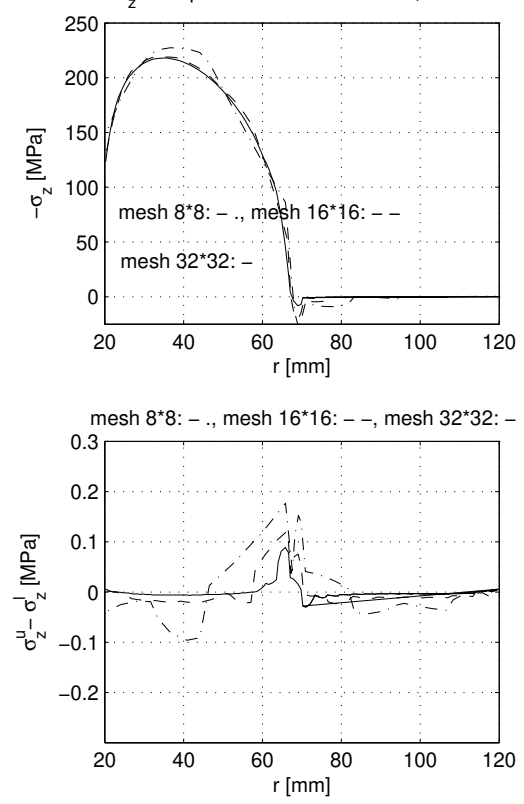

Figure 6. Task 1: solution with $h$-version, polynomial order for the displacements and stresses is $p=2$; a) mesh $8 \times 8$; b) normal stress $\sigma_{z}$ in the punch (upper body); c) distributions of normal stress $\sigma_{z}$, contact pressure $p_{n}$ and differences between the normal stresses of the upper $\left(\sigma_{z}^{u}\right)$ and lower $\left(\sigma_{z}^{l}\right)$ bodies for different meshes

The Reissner functional is applied and the same degree of polynomials are used for the approxination of the displacements and stresses: i.e. $\mathrm{npu}=\mathrm{nps}=p$. The fixed mesh contains 7 columns and 5 rows $($ mesh $7 \times 5$ ). Figure $8 \mathrm{a}-\mathrm{g}$ illustrate the results with polynomial order $p=8$ for the different stress components.

Figure 9 shows different components of the results: the normal stress $\sigma_{z}$, the contact pressures $p_{n}$, the difference between the normal stresses $\sigma_{z}^{u}$ and $\sigma_{z}^{l}$ (computed on the 
a)

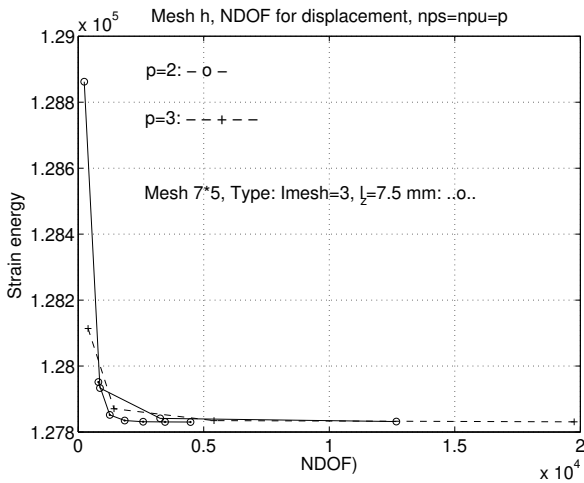

b)

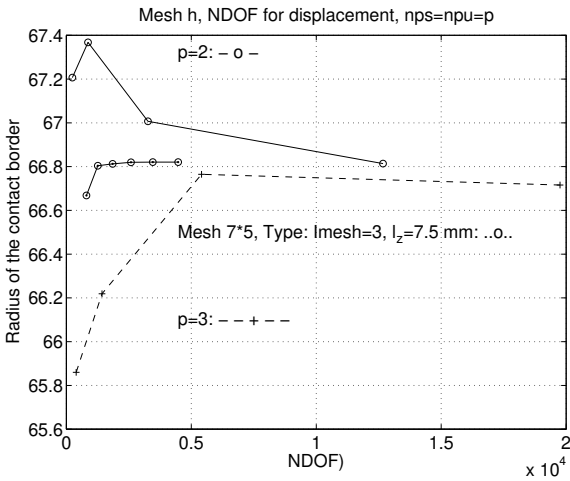

Figure 7. Convergence diagrams for $h$-version calculation with $p=2$ and $p=3($ mesh $4 \times 4,8 \times 8,16 \times 16,32 \times 32)$ and mesh $7 \times 5$ (identified by Type: Imesh $=3, l_{z}=7.5 \mathrm{~mm}$ ); a) for strain energy in

$\mathrm{Nmm}$; b) for contact border radius $r_{p}$ in $\mathrm{mm}$

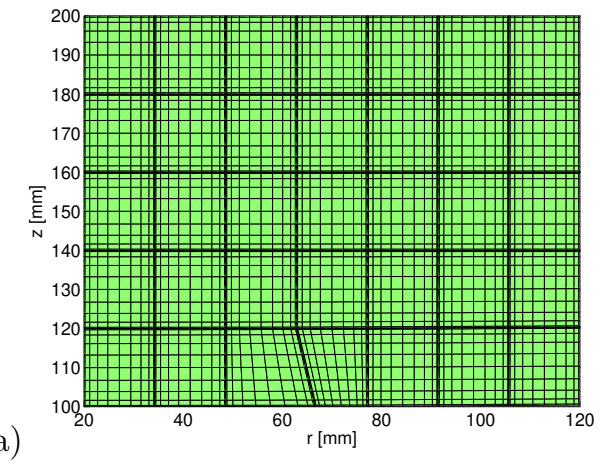

b)

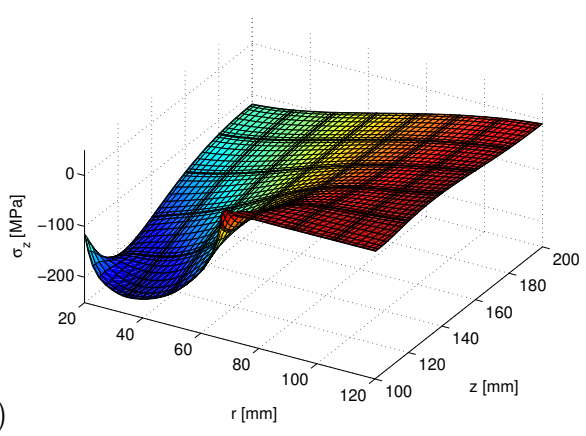

Figure 8. Solution of contact problem (Task 2 for body $B_{1}$ ) with uniform mesh $7 \times 5$, Imesh $=0$ : a) Final mesh after positioning, $\left.r_{p}=66.6168 \mathrm{~mm} ; \mathrm{b}\right)$ vertical normal stresses

upper and the lower bodies) with polynomial order $p=8$, and the distribution of $\sigma_{z}^{u}$ as a function of $r \geq r_{p}$ with different polynomial orders $(p=2, p=6$ and $p=8)$.

Oscillations can also be observed here, but it much smoother than in Task 1, where only $h$-extension elements were used (compare to Figure. 6a). In the zone of gap (a certain distance from the border of the contact zone) the boundary condition $\sigma_{z}^{u}=0$ is satisfied better with $p$-extension elements than with $h$-extension ones (compare Figure 6 to Figure 9). 

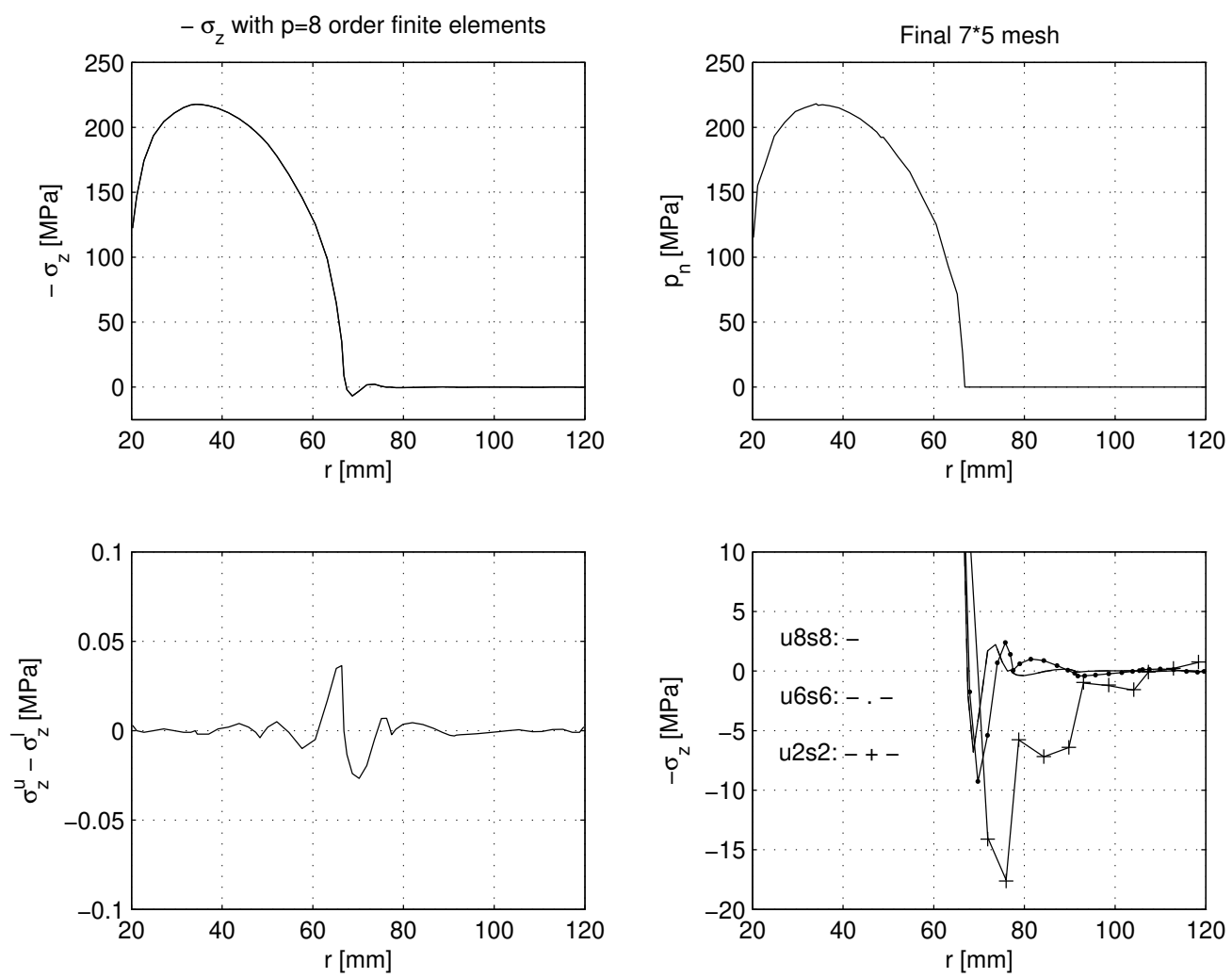

Figure 9. Solution of the contact problem with mesh type Imesh $=0$, computed by using different polynomial orders: $p=2(u 2 s 2), 6(u 6 s 6)$ and $8(u 8 s 8)$

6.3. Task 3. Choosing the appropriate finite element mesh is an essential step to achieve high accuracy. In the mesh type Imesh $=0$ there are equivalent elements. In the case of Imesh $=1$ the initial Imesh $=0$ is modified, i.e. near the border of the contact zone smaller elements are generated because the border point is a singular stress point.

The mesh $(7 \times 5)$ is applied with narrow elements on the right and left sides of the contact border point, i.e. single element layers are used on both sides of the border point in horizontal direction. The radial coordinate of the border point is $r_{p}$. The outer radius of the cylinder $r_{u}$ and the length of the separation zone is $l_{p u}=r_{u}-r_{p}$. The sizes of the elements around of border point are $\Delta_{r l}=l_{p u} f^{2}, \Delta_{r 2}=l_{p u} f$ where $f=0.125$. 
In vertical direction the size of the elements is increased gradually starting from the contacting elements and given by the following values for body $B_{1}$. In vertical direction there is a distance $l_{z}$ and the sizes of the elements are $\Delta_{z 1}=l_{z} f^{2}, \Delta_{z 2}=l_{z} f$ and in this $z$ direction the border of the next element is at $l_{z}$. The mesh for body $B_{2}$ is symmetrical to the $x$-axis.

Because the position $r_{p}$ is determined with iteration, the length $l_{p u}=r_{u}-r_{p}$ can change. The iteration is finished when the contact pressure at the border point reaches its minimun value $I_{1}=\min \left|c_{n} d(u)\right| \leq 0.0001$. In the computations we have three different values for $l_{z}=7.5,15$ and $20 \mathrm{~mm}$.

Imesh $=1$ : Mesh $(7 \times 5)$ is used with single element layer in horizontal direction on the left and the right hand side of the border point $\left(\Delta_{r l}=l_{p u} f^{2}\right)$.

Imesh $=2$ : Mesh $(7 \times 5)$ is applied with single element layer in horizontal direction on the left hand side of the border point $\left(\Delta_{r l}=l_{p u} f^{2}\right)$, but on the right hand side of the border point the mesh is finer because of the double element layer $\left(\Delta_{r 1}=l_{p u} f^{2}, \Delta_{r 2}=l_{p u} f\right)$.

Imesh $=3$ : Mesh $(7 \times 5)$ is used with double element layer on both the left and right hand side of the contact border point $\left(\Delta_{r 1}=l_{p u} f^{2}, \Delta_{r 2}=l_{p u} f\right)$.

Firstly, let us compare the simulations of Imesh $=1$ and Imesh $=2$. The results are practically the same, see Figure 10 . In Figure 10 c the change of mesh $7 \times 5$ is shown, according to the $r p$-version of the finite element method. The initially uniform mesh $7 \times 5$ is modified to (Figure $10 \mathrm{c}$ ) and then the final mesh (Figure $10 \mathrm{c}$ ) was obtained after 10 iteration steps.

Secondly, the solution of the contact problem with Imesh $=3$ is visualized by Figure 11. In Figure 12 one can see the results with original mesh (i.e. without positioning), and Figure 13 demonstrates the results using the node positioning (i.e. using the $r p$-extension method). It can also be realized that the oscillation of normal stress $\sigma_{z}$ at the border point of contact zone is very small compared to simulation results received by $p$-extension finite elements $($ Imesh $=0)$, see Figure 14

It is interesting that the distribution of the normal stress $\sigma_{z}$ in the contact zone is smooth enough even in the case of using the original mesh. The discrepancy between the contact pressure and normal stress theoretically should be equal to zero, in this aspect the positioning technique gives better results, as is shown in Figure 12 and Figure 13 .

Figure 15 shows different convergence diagrams: Figure $15 \mathrm{a}$ and $15 \mathrm{~b}$ demonstrate the convergence of the position of contact border $\left(r_{p}\right)$. It is clear that the $r p$-version of the computations is better than the $p$-version one.

We define the following error indicators using equations (5.5) and 5.6

$$
\begin{gathered}
I_{3 u}=I_{3}(\boldsymbol{u}), \quad I_{3 s}=I_{3}(\boldsymbol{T}), \\
I_{4 u}=I_{4}(\boldsymbol{u}), \quad I_{4 s}=I_{4}(\boldsymbol{T}), \\
\left(\operatorname{Error}_{F}\right)_{u}=\frac{100 I_{3 u}}{F_{z}}, \quad\left(\operatorname{Error}_{F}\right)_{s}=\frac{100 I_{3 s}}{F_{z}},
\end{gathered}
$$


where the resultant contact force is

$$
F_{z}=-\int_{S_{c}} \sigma_{n}^{(1)} \mathrm{d} S, \quad \sigma_{n}^{(i)}=\boldsymbol{n}^{(i)} \cdot \boldsymbol{T}^{(i)} \cdot \boldsymbol{n}^{(i)} .
$$
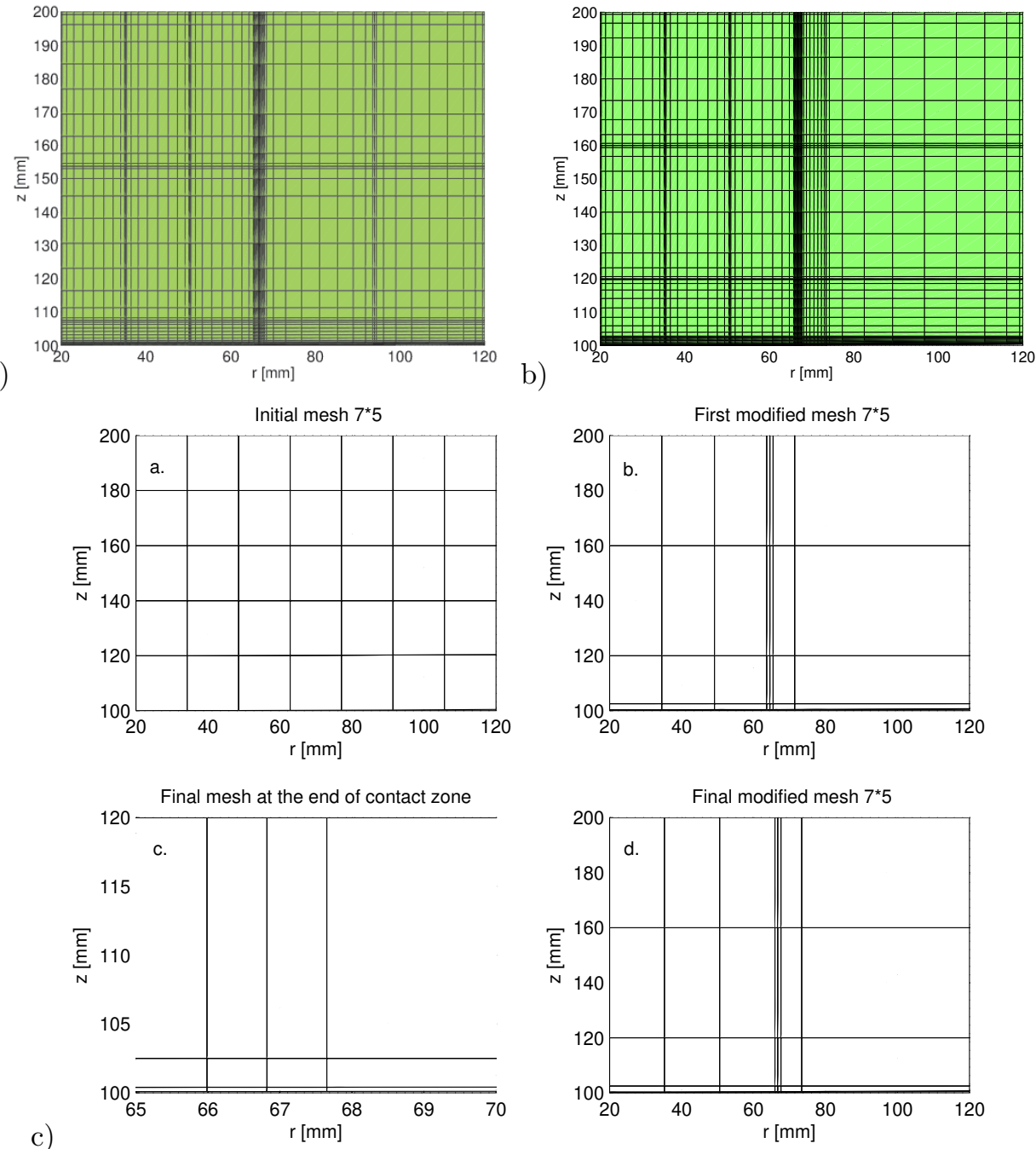

Figure 10. Solution with modified $\operatorname{Imesh}=1$, a) $l_{z}=7.5 \mathrm{~mm}, r_{p}=$ $66.8220 \mathrm{~mm}$; b, c) Imesh $=2, l_{z}=20.0 \mathrm{~mm}, r_{p}=66.8197 \mathrm{~mm}$ 
a)
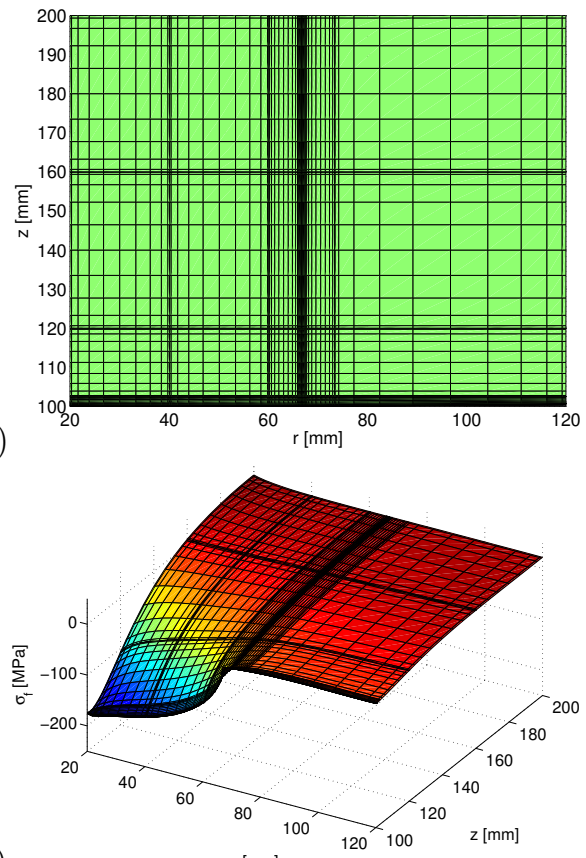

c)

$r[\mathrm{~mm}]$

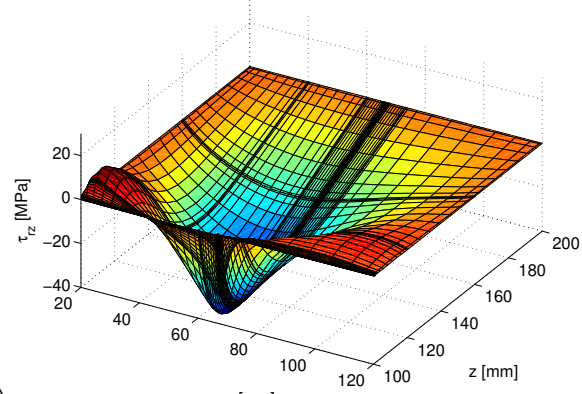

e) $r[\mathrm{~mm}]$ b)

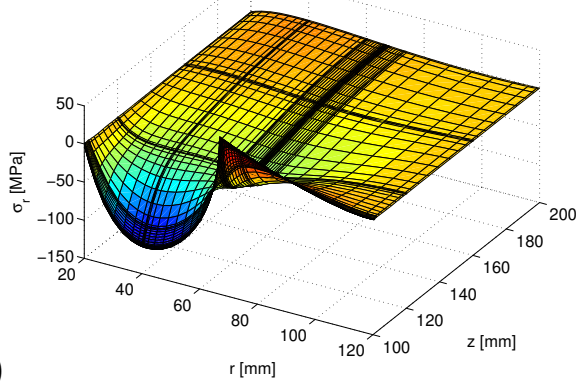

d)
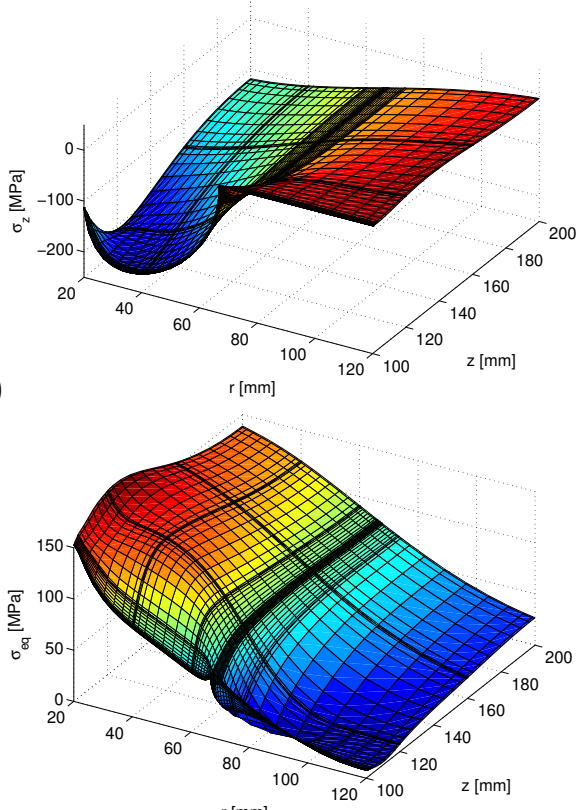

f)

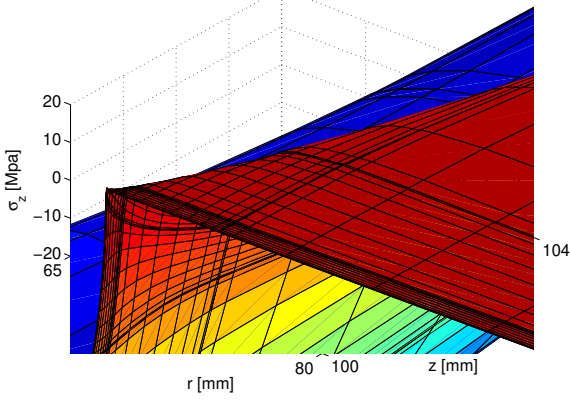

Figure 11. Solution of contact problem (Task 3 for body $B_{1}$ ) with small elements around contact border mesh $(7 \times 5)\left(\operatorname{Imesh}=3, l_{z}=\right.$ $20 \mathrm{~mm}, r_{p}=66.8262 \mathrm{~mm}$ ): a) Final mesh after positioning; b) $-\mathrm{d}$ ) radial, circumferential and vertical normal stresses; e) shear stress; f) Mieses equivalent stress; g) normal stress $\sigma_{z}$ near the border of the contact zone. The polynomial order for displacements and stresses is $p=8$ 

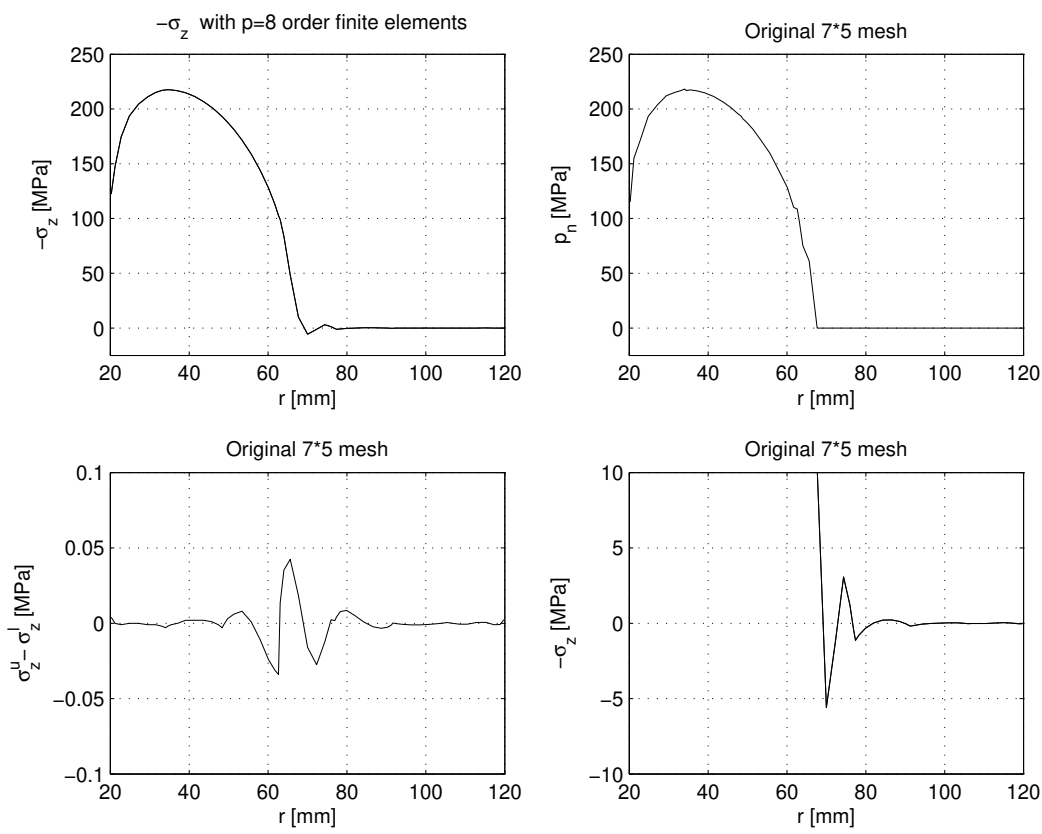

Figure 12. Contact stress distribution using initial mesh (contact pressure has large oscillation around the border of contact zone).

Figure 15 and $15 \mathrm{~d}$ provide a good comparison of the results obtained for the resultant force $F_{z}$. The $r p$-version has very good convergence. In these diagrams the results obtained by the use of the total potential energy functional are also displayed. It is surprising that the Reissner variational principle was not proved to be superior to the principle of minimum total potential energy, though displacements and stresses as two independent unknown fields have been approximated. Due to higher computational effort of the Reissner variational principle one can conclude that it is not worth applying it to similar contact problems.

It is also investigated what the connection is between the polynomial order of the stress and the polynomial order of the displacement fields. Three variants: Variant 1 (V1), Variant 2 (V2) and Variant 3 (V3) are analyzed:

$\mathrm{V} 1: \mathrm{nps}=\mathrm{npu}-1$;

$\mathrm{V} 2: \mathrm{nps}=\mathrm{npu}$;

$\mathrm{V} 3: \mathrm{nps}=\mathrm{npu}+1$,

where nps means the polynomial order of stress approximation function, and the polynomial order of displacement approximation function is denoted by npu. Figure 16 makes the comparison easier. 

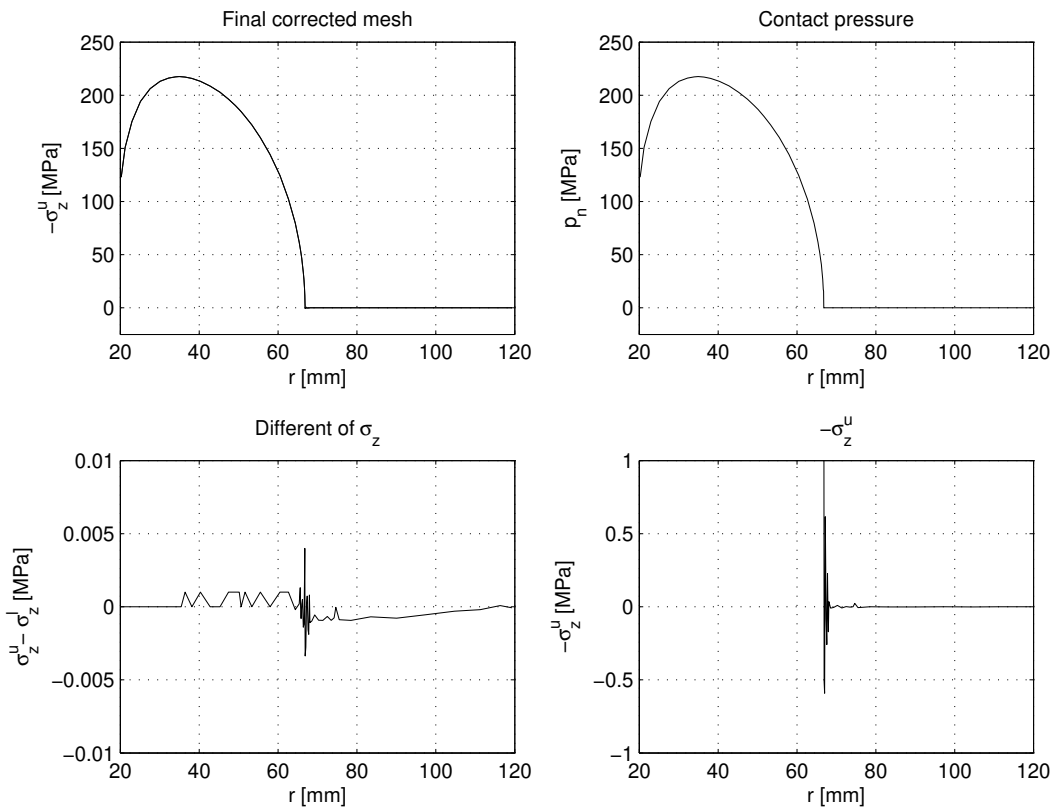

Figure 13. Contact stress distribution using the final mesh, Imesh $=$ $3, l_{z}=20 \mathrm{~mm}$

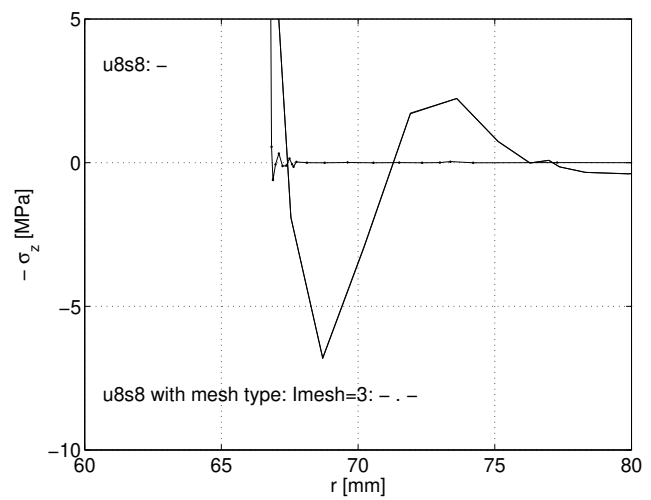

Figure 14. Oscillation of the normal stress around border point of contact zone $\left(\right.$ Imesh $=0:-$, Imesh $\left.=3:-.-, l_{z}=20 \mathrm{~mm}\right)$

Using the principle of minimum total potential energy with Imesh $=3, l_{z}=7.5 \mathrm{~mm}$ (see Table 1). 
a)
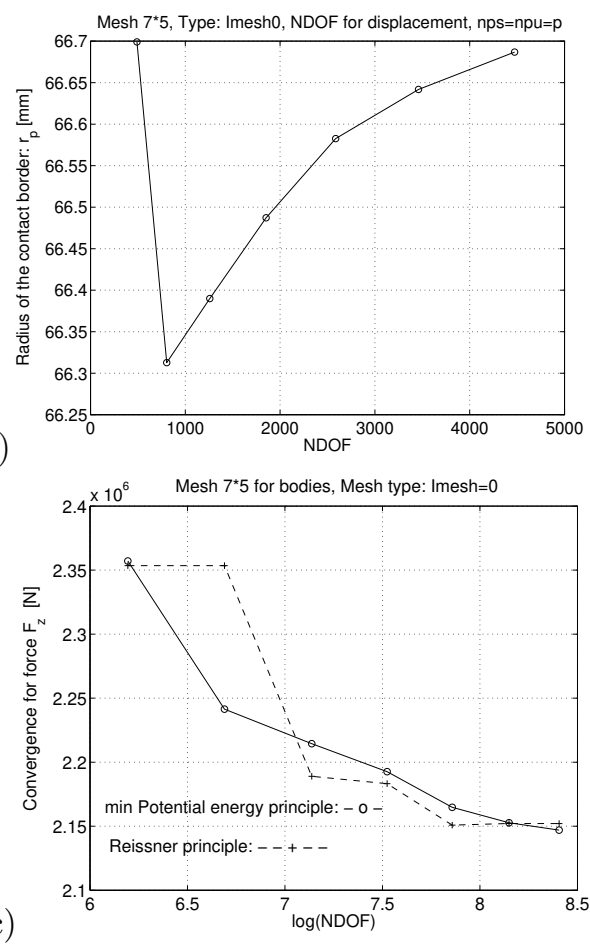

b)
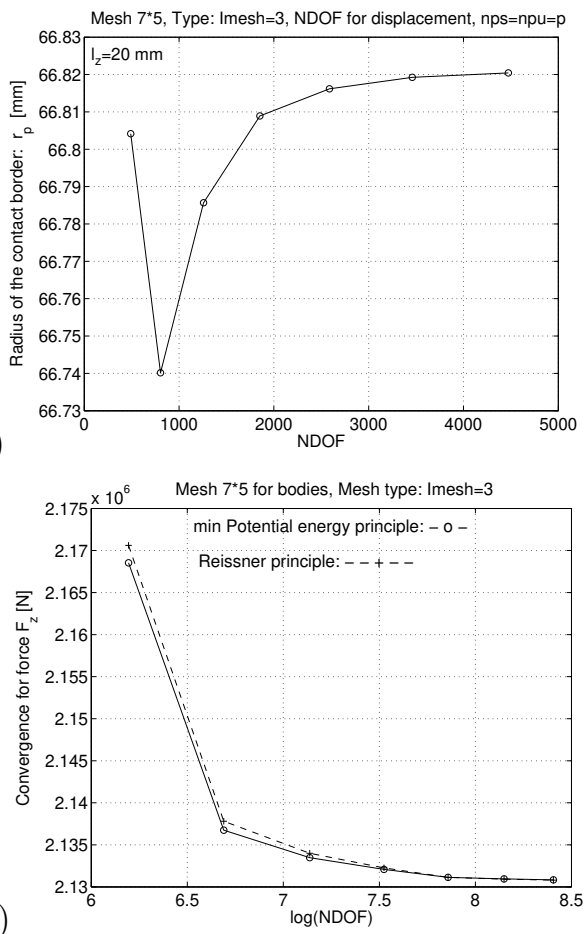

Figure 15. Convergence diagram for radius $r_{p}$ a) uniform mesh $($ Imesh $=0) ; b)$ meshed with small elements around border point of contact zone $(\operatorname{Imesh}=3)$. Resultant force $F_{z}$ c) Imesh $=0 ; \mathrm{d}$ ) Imesh $=2, l_{z}=7.5 \mathrm{~mm}$

The Reissner principle is applied to solve the same contact problem and using the same $r p$-version finite element mesh with increasing polynomial order $(\mathrm{npu}=\mathrm{nps}=p)$ and the results are summarized in Table 2 .

We can compare the results obtained by the total potential energy functional and Reissner principle. Practically the radius of the contact border is the same, the error indicators $I_{3}$ and $I_{4}$ are mainly smaller with the Reissner principle. The difference in resultant force $F_{z}$ is very small. The strain energy of Reissner principle is computed from the approximated stress field. The convergence rate of the strain energy is a bit larger for the Reissner principle.

It is evident that using the $(7 \times 5)$ mesh the solution gives a small discrepancy when the polynomial order changinges from $p=2$ to $p=8$. However, the relative error for $r_{p}$ is smaller than $0.2 \%$ and for $F_{z}$ this error shows $2 \%\left(\left[F_{z}(p=2)-F_{z}(p=\right.\right.$ $\left.8)] / F_{z}(p=8)\right)$.

Three mesh types $(\operatorname{Imesh}=1, \operatorname{Imesh}=2$ and $\operatorname{Imesh}=3)$ are used in order to compare the results for these two minimization principles $\left(l_{z}=7.5 \mathrm{~mm}\right)$. Results are collected in Table 3 for $\mathrm{nps}=\mathrm{npu}=p=8$ and $\mathrm{NDOF}=4474$. 

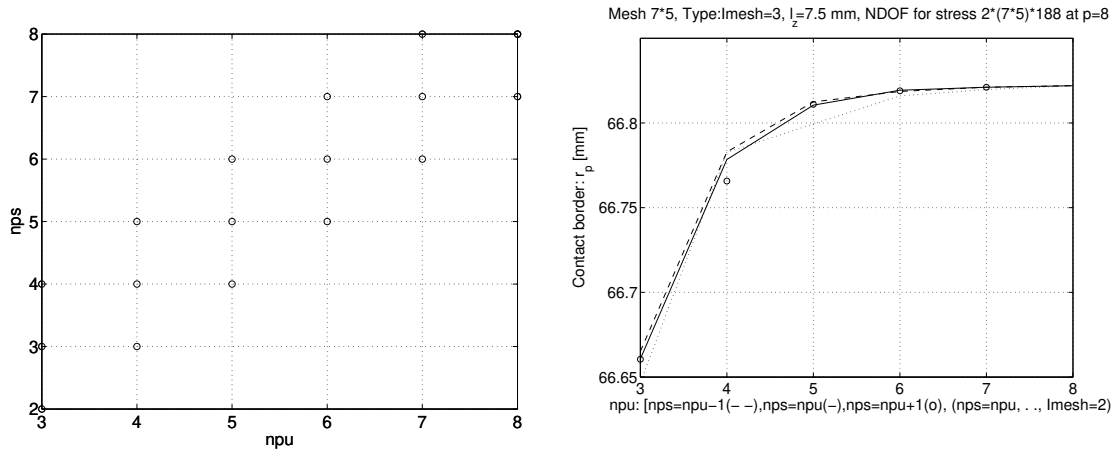

Figure 16. a) Change in polynomial order for displacements (npu) and for stresses (nps); b) Convergence diagram for border point of contact zone $r_{p}$ when mesh is modified by small elements $(\operatorname{Imesh}=3)$

Table 1. Results are summarized as a function of polynomial order $p$, based on total potential energy.

\begin{tabular}{rrrrrrr}
\hline$p$ & NDOF & $r_{p}[\mathrm{~mm}]$ & Pot.e $[\mathrm{Nmm}]$ & $F_{z}[\mathrm{~N}]$ & $I_{3}[\mathrm{~N} / \mathrm{mm}]$ & $I_{4}[\mathrm{~N} / \mathrm{mm}]$ \\
\hline 2 & 490 & 66.6759 & 128390.922 & 2168530.63 & 543.85 & 280.02 \\
3 & 804 & 66.6806 & 127940.062 & 2136748.84 & 119.48 & 110.73 \\
4 & 1258 & 66.7532 & 127865.300 & 2133480.24 & 57.14 & 59.29 \\
5 & 1852 & 66.7987 & 127840.504 & 2132085.58 & 35.23 & 43.61 \\
6 & 2586 & 66.8130 & 127831.901 & 2131137.97 & 22.11 & 36.77 \\
7 & 3460 & 66.8228 & 127830.607 & 2130957.02 & 21.53 & 34.86 \\
8 & 4474 & 66.8225 & 127830.353 & 2130835.13 & 16.81 & 32.46 \\
\hline
\end{tabular}

Table 2. Results are summarized as a function of polynomial order $p$, based on Reissner principle.

\begin{tabular}{rrrrrrr}
\hline$p$ & NDOF & $r_{p}[\mathrm{~mm}]$ & Strain.e $[\mathrm{Nmm}]$ & $F_{z}[\mathrm{~N}]$ & $I_{3}[\mathrm{~N} / \mathrm{mm}]$ & $I_{4}[\mathrm{~N} / \mathrm{mm}]$ \\
\hline 2 & 490 & 66.6632 & 128443.511 & 2170611.24 & 614.04 & 274.43 \\
3 & 804 & 66.6659 & 127951.403 & 2137806.80 & 132.08 & 89.02 \\
4 & 1258 & 66.7649 & 127871.373 & 2133994.69 & 70.21 & 53.16 \\
5 & 1852 & 66.8107 & 127843.167 & 2132259.59 & 37.73 & 42.50 \\
6 & 2586 & 66.8194 & 127832.525 & 2131129.16 & 18.51 & 34.05 \\
7 & 3460 & 66.8216 & 127830.756 & 2130920.19 & 14.92 & 32.07 \\
8 & 4474 & 66.8222 & 127830.400 & 2130804.34 & 11.77 & 30.88 \\
\hline
\end{tabular}

Figure 18 shows the results for $7 \times 5$ mesh, at $l_{z}=7.5,15$ and $20 \mathrm{~mm}$, and at Imesh $=1,2,3$, by $p=8$, when using the Reissner principle. It can be observed that the results are very similar. The smallest strain energy is supplied by Imesh $=3$ and $l_{z}=15 \mathrm{~mm}$. The minimum value for the contact border radius $r_{p}$ is with Imesh $=1$ and $l_{z}=15 \mathrm{~mm}$. Indicator $I_{3}$ is the smallest with Imesh $=2$ and $l_{z}=7.5 \mathrm{~mm}$, and $I_{4}$ is the smallest with Imesh $=3$ and $l_{z}=7.5 \mathrm{~mm}$. 
a)
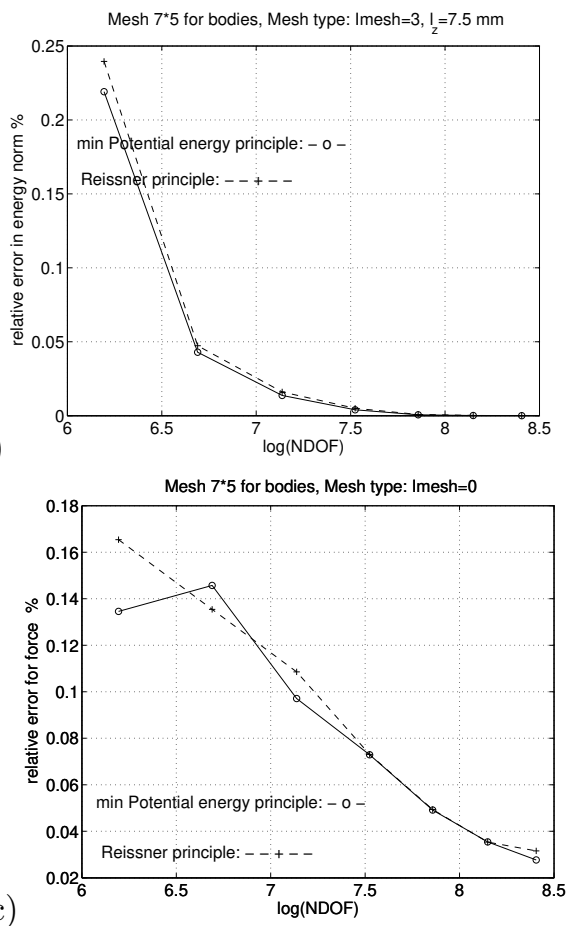

b)
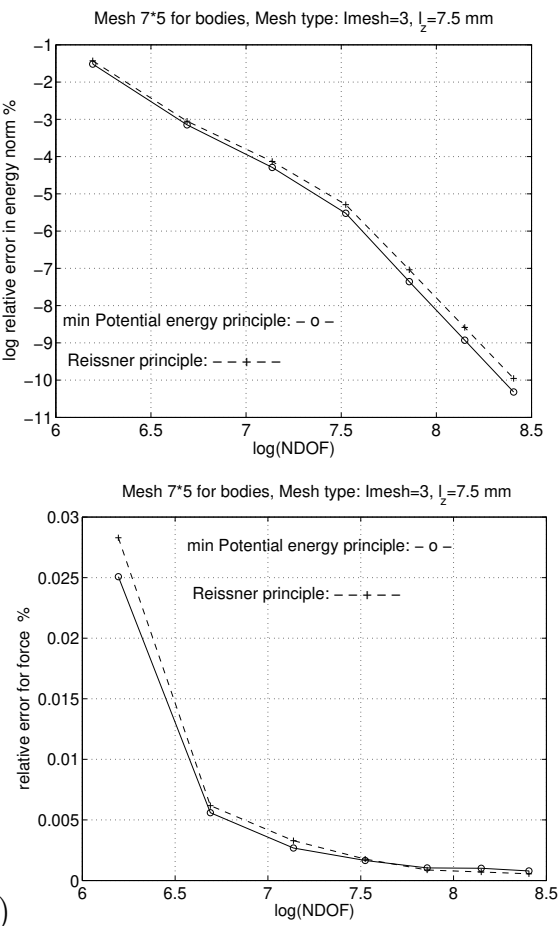

Figure 17. Comparing the convergence of the energy for finite element codes using minimum of the total potential energy and Reissner principle: a) relative error in the energy norm; b) logarithm of the relative error; c) Imesh $=0,\left(\operatorname{Error}_{F}\right)_{u}:-\circ-,\left(\operatorname{Error}_{F}\right)_{s}:-+-$; d) $\operatorname{Imesh}=3,\left(\text { Error }_{F}\right)_{u}:-\circ-,\left(\text { Error }_{F}\right)_{s}:-+-$

The relative difference between the maximum force and the minimum force in $F_{z}$ is less than $0.13 \%$.

Solving the above contact problem with the Reissner principle by mesh $8 \times 5$ (NDOF $=$ 5 090), the following results are obtained:

$$
\begin{array}{lllll}
\text { Imesh }=2 & l_{z}=7.5 \mathrm{~mm} & r_{p}=66.8221 \mathrm{~mm} & l_{z}=15 \mathrm{~mm} & r_{p}=66.8207 \mathrm{~mm} \\
\text { Imesh }=3 & l_{z}=7.5 \mathrm{~mm} & r_{p}=66.8223 \mathrm{~mm} & l_{z}=15 \mathrm{~mm} & r_{p}=66.8208 \mathrm{~mm}
\end{array}
$$

This indicates that the finer mesh $8 \times 5$ does not give a better or different solution, therefore applying mesh $7 \times 5$ gives sufficently accurate results using Imesh $=2$ according to Table 3 .

\section{EXAMPLE FOR HERTZ PROBLEM WITH FRICTION}

The contact problem for a truncated sphere with radius $R=800 \mathrm{~mm}$ and a cylinder with outer radius $r_{u}=10 \mathrm{~mm}$ is investigated. The truncated sphere is the upper body denoted by $B_{1}$, the cylinder is the lower one denoted by $B_{2}$. The height of 
a)
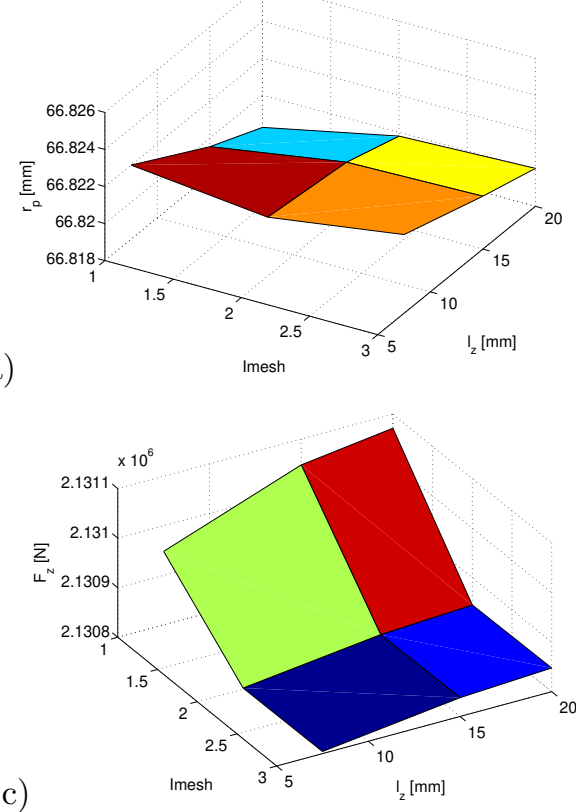

b)

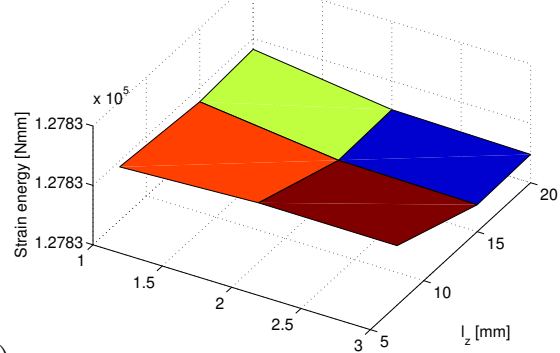

d)

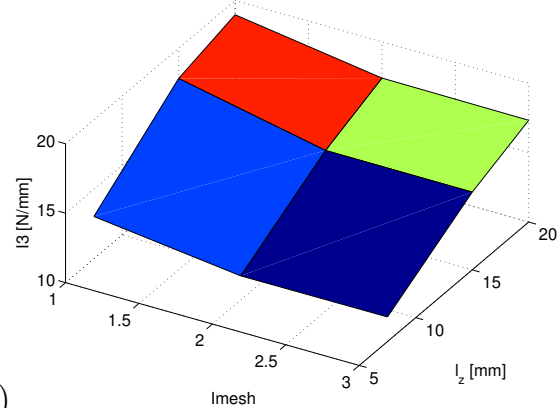

Figure 18. Results with mesh $7 \times 5$, Imesh $=1,2,3$ and $l_{z}=$ $7.5,15,20 \mathrm{~mm}$ by using the Reissner principle

the truncated sphere is $5 \mathrm{~mm}$ and its outer surface is a cylinder mantle with radius $10 \mathrm{~mm}$. Height of the lower cylinder is equal to $5 \mathrm{~mm}$.

Material parameters for the lower body are Young's modules $E_{2}=200 \mathrm{GPa}$ and Poisson ratio $\nu=0.3$. Sphere is regarded to be rigid and its Young's modules is given as $E_{1}=1000 E_{2}$. Vertical displacement $u_{z}=-w_{0}=0.005 \mathrm{~mm}$ is prescribed on the top surface of the body $B_{1}$. The coefficient of friction is $\mu=0.2$.

The initial mesh is shown in Figure 19 a. The modified mesh after solution of the frictional contact problem is given in Figure $19 \mathrm{~b}$. The $3^{\text {rd }}$ element has new nodal point positions, one of them is moved left to border of stick-slip region, and the other one is moved to right to the effective contact border.

The obtained distributions of the shear stresses along the contact domain are shown in Figure 20 for different load levels. In those points, where $\tau_{n}=\tau_{r z} \leq \mu p_{n}$ stick takes place, while in the rest of points, where $\tau_{n}=\tau_{r z}=\mu p_{n}$ here are slips. Distribution of the normal stress along the boundary $z=5$ and $z=10 \mathrm{~mm}$ of the sphere is displayed in Figure 21. The resultant vertical forces of both surfaces theoretically should be equal to each other. This requirement is fulfilled with an acceptably small error, they are equal up to the first three digits.

Penalty parameter in the tangential direction is $c_{\tau}=100 E_{2}$, and in the vertical (normal) direction it is $c_{n}=1000 E_{2}$. 
Table 3. Comparing results for different meshes $\left(l_{z}=7.5 \mathrm{~mm}\right)$.

\begin{tabular}{cccc}
\hline Imesh & $r_{p}[\mathrm{~mm}]$ & using Pot.e. min. princ. & using Reissner var. princ. \\
\hline 1 & & 66.8228 & 66.8220 \\
2 & & 66.8174 & 66.8219 \\
3 & & 66.8225 & 66.8222 \\
\hline Imesh & Strain e. $[\mathrm{Nmm}]$ & using Pot.e. min. princ. & using Reissner var. princ. \\
1 & & 127830.352 & 127830.38 \\
2 & & 127830.353 & 127830.40 \\
3 & & 127830.353 & 127830.40 \\
\hline Imesh & $F_{z}[\mathrm{~N}]$ & using Pot.e. min. princ. & using Reissner var. princ. \\
1 & & 2130904.48 & 2130948.39 \\
2 & & 2130860.09 & 2130802.48 \\
3 & & 2130835.13 & 2130804.34 \\
\hline Imesh & $I_{3}[\mathrm{~N} / \mathrm{mm}]$ & using Pot.e. min. princ. & using Reissner var. princ. \\
1 & & 17.49 & 13.04 \\
2 & & 12.26 & 11.75 \\
3 & & 16.81 & 31.67 \\
\hline Imesh & $I_{4}[\mathrm{~N} / \mathrm{mm}]$ & using Pot.e. min. princ. & using Reissner var. princ. \\
1 & & 33.09 & 30.89 \\
2 & & 31.92 & 30.88 \\
3 & & 32.46 &
\end{tabular}

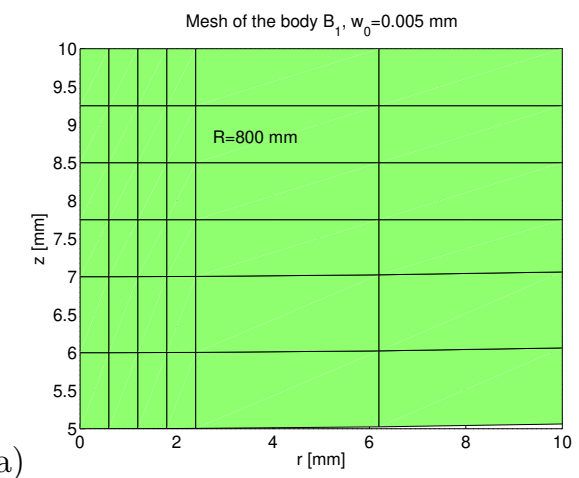

b)

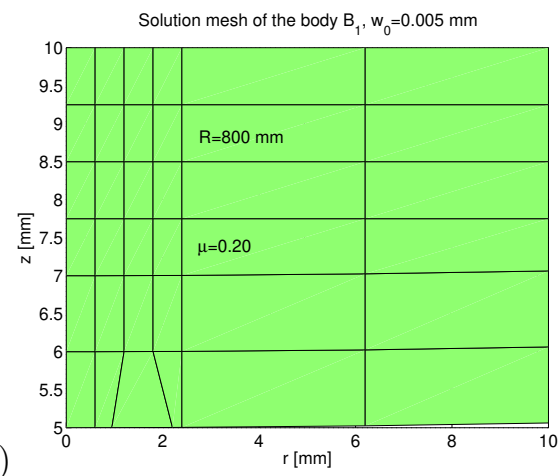

Figure 19. Meshes of body $B_{1}$ : a) original b) at the end of solution of contact problem using position technique. The stick-slip border is at $r_{s t-s l}=0.947 \mathrm{~mm}$, the effective contact border is at $r_{p}=2.203 \mathrm{~mm}$

The value of the friction coefficient $\mu$ essentially influences the length of stick zone. The results of the tangential stresses obtained for three different frictional coefficient are shown in Figure 22. However $\mu$ has only a little influence on contact border radius $r_{p}$ as given here: $r_{p}(\mu=0.1)=2.213, r_{p}(\mu=0.2)=2.203, r_{p}(\mu=0.3)=2.193 \mathrm{~mm}$. 


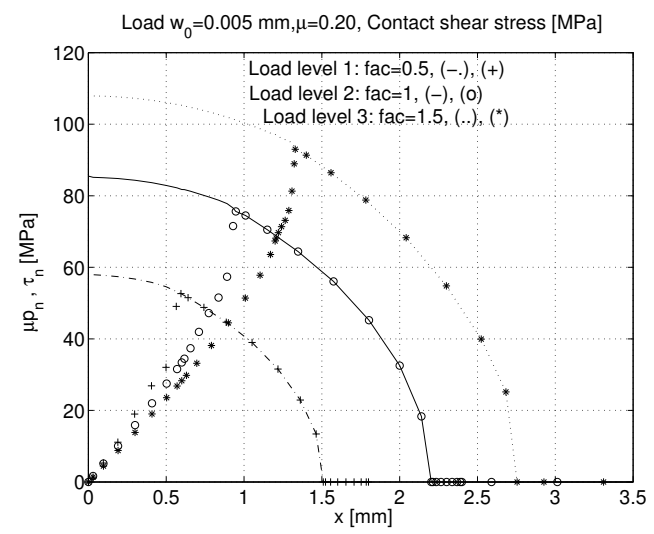

Figure 20. Shear stresses along the contact surface

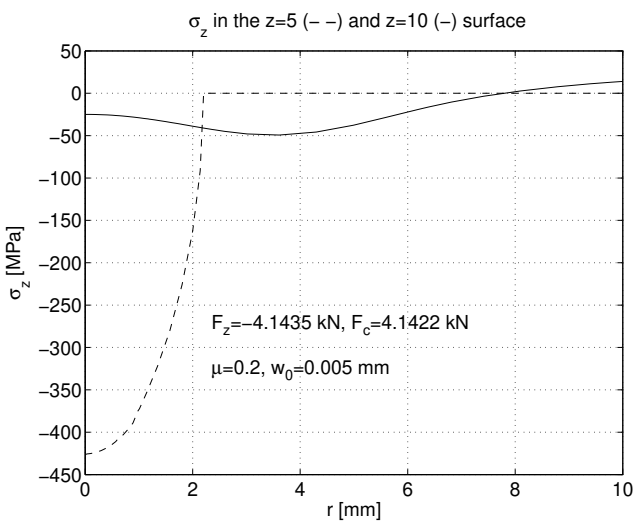

Figure 21. Distribution of $\sigma_{z}$ along the contact surfaces at $z=5$ and $z=10 \mathrm{~mm}$ where the vertical displacement is prescribed

The contact pressure maximums are 423.83, 427.49, 429.13 MPa at different friction coefficients, $\mu=0.1,0.2,0.3$, respectively.

Finally problems are analyzed when only the rigidity of the sphere is changed.

The results are collected in Table 4. In order to compare the results of task 4 in Table 4 with Hertz theory, the maximum of contact pressure $p_{0}$ and resultant load $F_{z}$ are calculated with $r_{p}(\mu=0.2)=a=2.232 \mathrm{~mm}$.

Using the Hertz formula the force $F_{z, \text { Hertz }}=4 E_{*} a^{3} /(3 R)=2.037 \mathrm{kN}$ and pressure $p_{0, H \text { ert } z}=\frac{3 F_{z, H e r t z}}{2 \pi a^{2}}=195.19 \mathrm{MPa}$ are calculated with $\frac{1}{E_{*}}=\frac{1-\left(v_{1}\right)^{2}}{E_{1}}+\frac{1-\left(v_{2}\right)^{2}}{E_{2}}$.

The relative errors are very small:

error $F_{z}=100 \frac{F_{z, \text { Hert } z}-F_{z}}{F_{z, \text { Hert } z}}=-0.736 \%, \quad$ error $p_{0}=100 \frac{p_{0, \text { Hert } z}-p_{0}}{p_{0, \text { Hert } z}}=-2.05 \%$. 


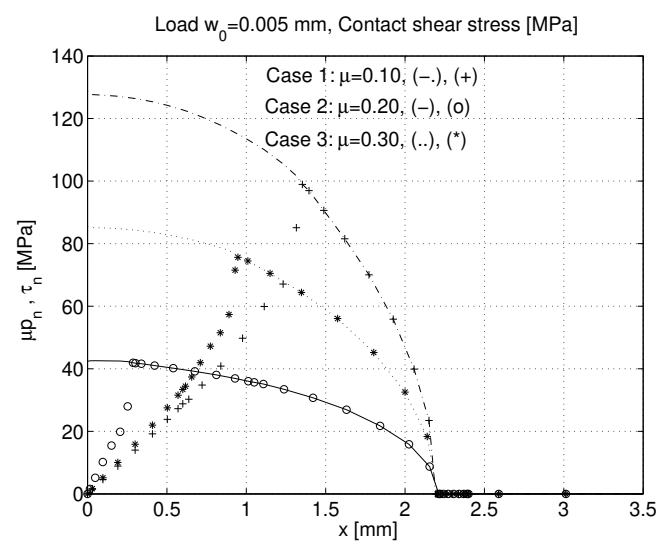

Figure 22. Shear stress along the contact surface at different coefficients of friction
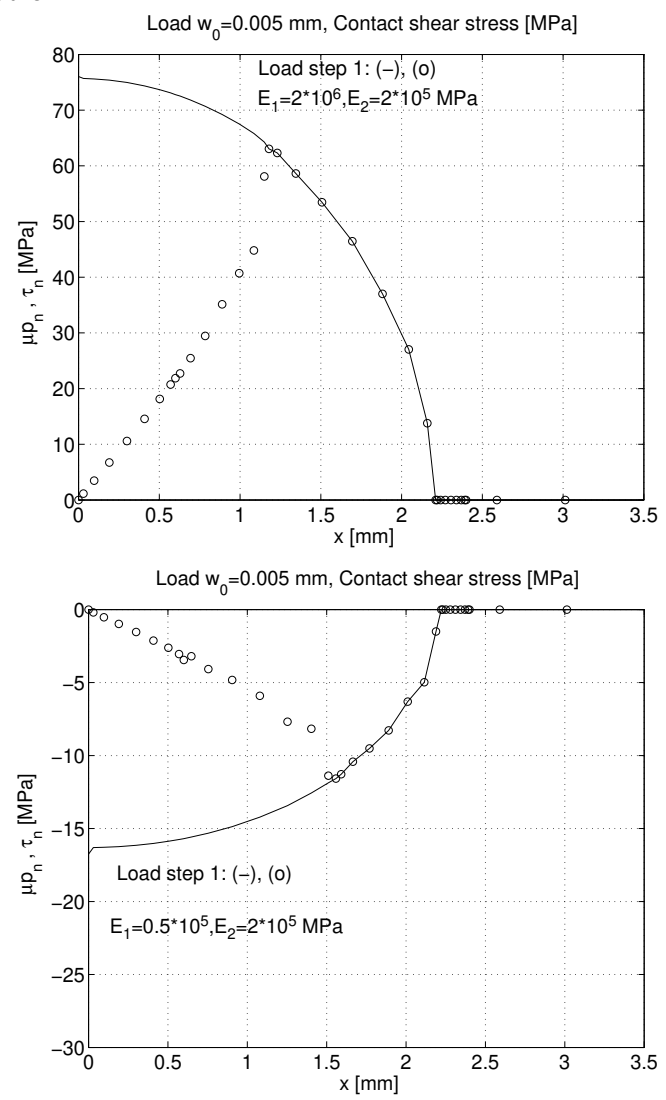

Figure 23. Shear stress distribution along the contact surface for different material parameters of the sphere, a) $\left.E_{1}=2000 \mathrm{GPa}, \mathrm{b}\right) E_{1}=$ $50 \mathrm{GPa}$ 
Table 4. Results of the Hertz problem for different friction coefficient

\begin{tabular}{ccccccc}
$\mu\left(\nu=\nu_{1}=\nu_{2}=0.3\right)$ \\
\hline $\begin{array}{c}\mu=0.2 \\
\text { Number of task }\end{array}$ & $\begin{array}{c}E_{1} \\
{[\mathrm{GPa}]}\end{array}$ & $\begin{array}{c}E_{2} \\
{[\mathrm{GPa}]}\end{array}$ & $\begin{array}{r}r_{s t-s l} \\
{[\mathrm{~mm}]}\end{array}$ & $\begin{array}{c}r_{p} \\
{[\mathrm{~mm}]}\end{array}$ & $\begin{array}{c}p_{0} \\
{[\mathrm{MPa}]}\end{array}$ & $\begin{array}{c}F_{z} \\
{[\mathrm{kN}]}\end{array}$ \\
\hline 1 & $\infty$ & 200 & 0.947 & 2.203 & 427.49 & 4.143 \\
2 & 2000 & 200 & 1.179 & 2.211 & 380.18 & 3.761 \\
3 & 1000 & 200 & 1.181 & 2.222 & 372.37 & 3.720 \\
$4 \mu=0$ & 200 & 200 & - & 2.232 & 199.19 & 2.052 \\
5 & 50 & 200 & 1.558 & 2.222 & 83.779 & 0.825 \\
\hline
\end{tabular}

In tasks 1-3 the tangential displacements of the sphere are smaller than of the cylinder, but the opposite was calculated for task 5 . This is due to the fact that the tangential stress $\tau_{n}=\tau_{r z}$ of the sphere is positive for tasks 1-3, and shear stress is negative for task 5 (see Figure 23).

\section{Conclusions}

Axially symmetric normal contact problems have been investigated by the $h$ version, $p$-version and $h p(r p)$-version finite element methods. The computations have been performed by a special purpose finite element package. The code can be applied for contact problems which leads to only a single connected contact zone.

In this paper the contact conditions are checked at the Gauss integration points. In order to obtain highly accurate results we use a special nodal positioning technique which ensures the contact along the whole edge of the contacting finite elements and very small oscillation in the gap zone.

Comparing the numerical results we concluded the following.

- When using equal number of degrees of freedom for $h$ - and $p$-versions the contact pressure distributions are similar. The maximum value of the contact pressure is bigger for $h$-version than for $p$-version. The boundary condition for the normal stress in the gap zone is satisfied better by $p$-version than by $h$-version.

- Accurate position of the contact border point can be determined by the use of the $h p(r p)$-extension finite elements with nodal positioning technique. The results are practically free of oscillations, and the dynamic boundary conditions are satisfied with high accuracy in the gap region as well.

- The convergence of the $h p(r p)$-version has been proved by numerical computations. Using finite element mesh with one or two layers of narrow elements at the contact border point and performing computations with a sequence of high values of $p(p=5,6,7,8)$ give a convergent series for the position of the contact border point.

- The accuracy of the solution with the Reissner principle is not better (at the same NDOF for displacement field) than the using minimum principle of potential energy. For engineering contact problems it is recommended to use the principle of minimum potential energy because of faster computation.

- Application of the special positioning technique for the frictional contact problem gives good accuracy in solutions. 
Acknowledgement. The present research was partially supported by the Hungarian Academy of Sciences, by grant NKFIH 115701.

\section{REFERENCES}

1. V. M. Fridman and V. S. Chernina. "Iteration methods applied to the solution of contact problems of bodies." Mehanika Tverdogo Tela AN, USSR, 1, (1967), pp. $116-120$.

2. I. Páczelt. "Iterational method applied to the solution of contact problems of elastic systems having elements in unilateral relation (in Russian)." Acta Technica Hungarorum, 76, (1974), pp. 217-241.

3. T. F. Conry and G. A. Seireg. "A mathematical programming method for design of elastic bodies in contact." ASME, Journal of Applied Mechanics, 38, (1971), pp. 387-392. DOI: 10.1115/1.3408787.

4. J. J. Kalker and Van Y. Randen. "A minimum principle for frictionless elastic contact problems." Journal of Engineering Mathematics, 6, (1972), pp. 193-206. DOI: $10.1007 / \mathrm{BF} 01535102$.

5. M. Fremond. "Dual formulation for potential and complementary energies. Unilateral boundary conditions application to the finite element method." The Mathematics of Finite Elements and Applications. Academic Press, 1973, pp. 175188. ISBN: 978-1483-2688-42. URL: https ://www . sciencedirect . com/book/ 9780127472508/the-mathematics-of-finite-elements-and-applications.

6. A. Frankavilla and O. C. Zienkiewicz. "A note on numerical computation of elastic contact problems." International Journal for Numerical Methods in Engineering, 9, (1975), pp. 913-924. DOI: 10.1002/nme.1620090410.

7. E. Haug, R. Chand, and K. Pan. "Multibody elastic contact analysis by quadratic programming." Journal of Optimization Theory and Applications, 21(2), (1977), pp. 189-198. DOI: $10.1007 /$ BF00932519.

8. I. Páczelt and B. Herpai. "Some remarks on the solution of contact problems of shells." Archiwum Budowy Maszyn XXIV, 1, (1977), pp. 117-202.

9. I. Páczelt. "Some remarks to the solution of quadratic programming problems." Publicatins of the Technical University Heavy Industry, Series D. Natural Sciences, 33, (1979), pp. 137-156.

10. Nguyen Dan Hung and Géry De Saxce. "Frictionless contact of elastic bodies by finite element method and mathematical programming technique." Computers and Structures, 11, (1980), pp. 55-67.

11. A. Klarbring. "A mathematical programming approach to three-dimensional contact problems." Computer Methods in Applied Mechanics and Engineering, 1985 (58,), pp. 175-200. DOI: 10.1016/0045-7825 (86) 90095-2.

12. G. Björkman. "The solution of large displacement frictionless contact problems using a sequence of linear complementary problems." International Journal for Numerical Methods in Engineering, 31 (1991), pp. 1533-1566. DOI: 10.1002/ nme.1620310808.

13. S. Chan and I. Tuba. "A finite element method for contact problems of bodies." International Journal of Mechanical Sciences, 13, (1971), pp. 615-639. DOI: 10. 1016/0020-7403(71)90033-6 
14. B. Fredrikson. "Finite element solution of surface nonlinearities in structural mechanics problems." Computers and Structures, 6, (1976), pp. 281-290.

15. I. Páczelt. "Solution of elastic contact problems by the finite element displacement method." Acta Technica Hungarorum, 82, (1976), pp. 353-375. URL: 193. $6.1 .94: 9080 /$ ?docId=28267.

16. T. R. J. Hughes and R. L. Taylor. "A finite element method for a class of contact impact problems." Computer Methods in Applied Mechanics and Engineering, 8, (1976), pp. 249-276. DOI: 10.1016/0045-7825(76)90018-9.

17. K. J. Bathe and A. Chaudhary. "A solution method for planar and axisymmetric contact problems." International Journal for Numerical Methods in Engineering, 21 (1985), pp. 65-88. DOI: 10.1002/nme.1620210107.

18. B. Nour-Omid and P. Wriggers. "A two level iteration method for solution of contact problems." Computer Methods in Applied Mechanics and Engineering, 54, (1986), pp. 131-144. DOI: 10.1016/0045-7825(86)90122-2.

19. A. B. Chaudhary and K. J. Bathe. "A solution method for static and dynamic analysis of three-dimensional contact problems with friction." Computers and Structures, 24(6), (1986), pp. 855-873.

20. J. Égert and J. Altenbach. "Inkrementelle Iterationsmethoden zur Lösung elastischer Kontaktaufgaben mit Reibung mittels spezieller isoparametrischer 2D- und 3D-kontaktelemente." Technische Mechanik, 10, (1989), pp. 120-137. uRL: http: //www15.ovgu.de/ifme/zeitschrift_tm/1989_Heft2/Egert.pdf.

21. J. E. Mottershead, S. K. Pascone, and R. G. English. "A general finite element approach for contact stress analysis." International Journal for Numerical Methods in Engineering, 33, (1992), pp. 765-779. DOI: 10.1002/nme.1620330407.

22. P. Papadopoulos and R. L. Taylor. "A simple algorithm for three- dimensional finite element analysis of contact problems." Computers and Structures, 46, (1992), pp. 1107-1118.

23. N. Kikuchi, J. T. Oden, and G. F. Carcey. Finite Elements: Special Problems in Solid Mechanics. Prentice Hall, Englewood, 1984.

24. J. C. Simo, P. Wriggers, and R. L. Taylor. "A perturbed Lagrangian formulation for the finite element solution of contact problems." Computer Methods in Applied Mechanics and Engineering, 50, (1985), pp. 163-180. DOI: 10.1016/00457825(85) 90088-X.

25. J. W. Ju and R. L. Taylor. "A perturbed Lagrangian formulation for the finite element solution of nonlinear frictional contact problems." Journal of Theoretical and Applied Mechanics, Special issue, Suplement No. 1., 7, (1988), pp. 1-14.

26. J. C. Simo and T. A. Laursen. "An augmented lagrangian treatment of contact problems involving friction." Computers and Structures, 42(7), (1992), pp. 97116.

27. T. A. Laursen and J. C. Simo. "A continuum-based finite element formulation for the implicit solution of multibody, large deformation frictional contact problems." International Journal for Numerical Methods in Engineering, 36, (1993), pp. 3451-3485. DOI: 10.1002/nme.1620362005

28. D. P. Berteskas. Constrained Optimization and Lagrange Multiplier Methods. 2nd edition. Academic Press, NewYork, 2014. DoI: 10.1016/C2013-0-10366-2. 
29. R. Fletcher. Practical Methods of Optimization. 2nd edition. John Wiley, NewYork, 1989.

30. Z. H. Zhong and J. Mackerle. "Static contact problems a review." Engineering Computations, 9 (1992), pp. 13-37. DOI: $10.1108 /$ eb023846.

31. U. Nackenhorst. An adaptive finite element method to analyse contact problems. In Contact Mechanics II, Computational Techniques. Ed. by M. H. Aliabali and C. Alessandri. Southampton, Boston: Computational Mechanics Publications, 1991, pp. 241-248.

32. P. Wriggers. Finite elements for thermomechanical contact and adaptive finite element analysis of contact problems. CISM Course: No. 388, Chapter 4 in New developments in contact problems. Ed. by P. Wriggers and P. Panatiotopoulos. Wien: Springer Verlag, 1999, pp. 179-246. DOI: 10.1007/978-3-7091-2496-3.

33. B. A. Szabó and I. Babuska. Finite Element Analysis. John Wiley \& Sons, New York, 1991.

34. C. Y. Lee and J. T. Oden. "Theory and approximation of quasistatic frictional contact problems." Computer Methods in Applied Mechanics and Engineering, 106, (1993), pp. 407-429. DOI: 10.1016/0045-7825(93) 90098-I.

35. U. Gabbert and K. Weinberg. "A pNh-element formulation for contact analysis." Zeitschrift für Angewandte Mathematik und Mechanik (ZAMM), 74(1), (1994), pp. 195-197. DOI: 10.1002/zamm.19940740407.

36. R. Buczkowski, M. Kleiber, and U. Gabbert. "On linear and higher order standard finite elements for 3d-nonlinear contact problem." Computers and Structures, 53, (1994), pp. 817-823.

37. I. Páczelt and T. Szabó. Application of the augmented lagrangian technique for solution of contact optimisation problems. Contact Mechanics II, Computational Techniques. Ed. by M. H. Aliabali and C. Alessandri. Southampton, Boston: WIT Press, 1995, pp. 249-256. DOI: 10.2495/CON950291.

38. I. Páczelt and A. Baksa. "Solution of contact problems using p-extension finite elements." Proceedings of XXXVII Summer School: Conference Advanced Problems in Mechanics. Ed. by D.A. Indeitsev and S. M. Kristov. 2009, pp. 507-519. URL: http://apm-conf .spg.ru

39. D. Franke, A. Dünster, V. Nübel, and E. Rank. "A computation of the h-, p-, hpand rp-version of the FEM for the solution of the 2D Hertzian contact problem." Computational Mecheanics, 45, (2010), pp. 513-522. DOI: 10 . 1007 /s00466009-0464-6.

40. A. Konyukhov and K. Schweizerhof. "Incorporation of contact for high-order finite element in convariant form." Computer Methods in Applied Mechanics and Engineering, 198, (2009), pp. 1213-1223. DOI: 10.1016/j.cma.2008.04.023.

41. D. Franke, E. Rank, and A. Dünster. "Computational contact mechanics based on the rp-version of the finite element method." International Journal of Computational Methods, 8, (2011), pp. 493-512. DOI: 10.1142/S0219876211002630.

42. I. Páczelt, B. A. Szabó, and T. Szabó. "Solution of contact problem using the hpversion of finite elements, accepted for publication." Computers $\&$ Mathematics with Applications, 38 (1999), pp. 49-69. DOI: 10.1016/S0898-1221(99)002618 
43. G. Duvat and J. L. Lions. Les Inequations en Mecanique et en Physique. Dunod, Paris, 1972.

44. I. Hlavacek, J. Haslinger, J. Necas, and J. Lovicek. Solution of Variational Inequalities in Mechanics, Applied Mathematical Sciences. Volume 66. Series of Springer Verlag, Berlin, 1980.

45. P. D. Panagiotopoulos. Inequality Problems in Mechanics and Applications: Convex and Nonconvex Energy Functions. Birkhaüser, Basel, 1985. DOI: 10.1007/ 978-1-4612-5152-1.

46. J. T. Oden and N. Kikuchi. Contact Problem in Elasticity: A Study of Variational Inequalities and Finite Element Methods. SIAM, Philadelphia, 1988.

47. J. I. Telega. "Variational principles for mechanical contact problems (in Russian)." Advances of Mechanics, 10, (1987), pp. 3-95.

48. J. Haslinger and P. Neittaanmaki. Finite Element Approximation for Optimal Shape Design. 2nd. John Wiley \& Sons, 1996.

49. J. Kalker. Three-dimensional Elastic Bodies in Rolling Contact. Academic Publisher, Dordrecht, 1990. DOI: 10.1007/978-94-015-7889-9.

50. M. Raous, P. Charband, and F. Lebon. "Numerical methods for frictional contact problems and applications." Journal of Theoretical and Applied Mechanics, Special Issue, Supplement No. 1, 7, (1988), pp. 111-128.

51. A. Klarbring. "Contact, friction, discrete mechanical structures and mathematical programming." CISM course: Contact problems: No. 388 Chapter 2, New developments in contact problem. Ed. by P. Wriggers and P. Panatiotopoulos. Wien: Springer Verlag, 1999, pp. 55-100. DOI: 10.1007/978-3-7091-2496-3.

52. S. Hilding, A. Klarbring, and J. Peterson. "Optimization of structures in unilateral contact." Applied Mechanics Reviews, 52(4), (1999), pp. 139-160. DOI: 10.1115/1.3098931

53. Y. Volpert. "Space Enrichment Methods for the Numerical Solution of Mechanical Contact Problems." PhD. Washington University, Department of Mechanical Engineering, St. Louis, 1995.

54. Y. Volpert, T. Szabó, I. Páczelt, and B. A. Szabó. "Application of the space enrichment method to problems of mechanical contact." Finite elements in Analysis and Design, 24, (1997), pp. 157-170. DOI: 10.1016/S0168-874X(96) 000479

55. I. Páczelt and T. Szabó. "Solution of contaqct optimization problems of cylindrical bodies using hp-FEM." International Journal for Numerical Methods in Engineering, 53, (2002), pp. 123-146. DOI: 10.1002/nme.395.

56. A. Baksa, I. Páczelt, and T. Szabó. "Solution of 3D contact problems using spline interpolation." Journal of Computational and Applied Mechanics, 9(2), (2014), pp. 125-147. DOI: 10.32973/jcam.2014.007.

57. V. A. Yastrebov. Numerical Mechanics in Contact Mechanics. John Wiley \& Sons Inc. Hoboken, 2013. DOI: 10.1002/9781118647974.

58. P. Wriggers. Computational Contact Mechanics. Springer, New York, 2006. DOI: 10.1007/978-94-015-9048-8.

59. A. Konyukhov and K. Schweizerhof. Computational Contact Mechanics. SpringerVerlag Berlin Heidelberg, 2013. DOI: 10.1007/978-3-642-31531-2. 
60. T.A. Laursen. Computational Contact and Impact Mechanics: Fundamentals of Modeling Interfacial Phenomena in Nonlinear Finite Element Analysis. SpringerVerlag, New York, 2003. DOI: 10.1007/978-3-662-04864-1.

61. P. Solin, K. Segeth, and I. Dolezel. Higher-Order Finite Element Methods. Chapman \& Hall/CRC, London, 2004.

62. L. Demkowicz, J. Kurtz, D. Pardo, M. Paszenski, W. Rachowicz, and A. Zdunek. Computing with hp-adaptive finite elements, Frontiers: Three Dimensional Elliptic and Maxwell Problems with Applications. Vol. 2nd. Chapman \& Hall/CRC, 2008.

63. I. G. Goryacheva. Contact Mechanics in Tribology. Springer Netherlands, 1998. DOI: 10.1007/978-94-015-9048-8.

64. I. Páczelt and Z. Mróz. "Solution of wear problems for monoton and periodic sliding with p-version of finite element method." Computer Methods in Applied Mechanics and Engineering, 1, (2012), pp. 249-252. DOI: 10.1016/j .cma.2012. 02.012 .

65. N. V. Banichuk and S. Yu. Ivanova. Optimal structural design. Walter de Gruyter GmbH, Berlin, 2017.

66. M. Raous, M. Jean, and J. J. Moreau, eds. Contact Mechanics. Springer US, NewYork, 1995. DOI: 10.1007/978-1-4615-1983-6.

67. J. A. C. Martins and M. O. P. Monteiro Marques, eds. Contact Mechanics, Proceedings of the 3rd Contact Mechanics International Symposium. Kluwer Academic Publishers, Dordrecht, 2002.

68. A. Popp and P. Wriggers. Contact Modeling for Solids and Particals. CISM International Centre for Mechanical Sciences 585. Springer International Publishing, 2018. DOI: 10.1007/978-3-319-90155-8.

69. P. Wriggers and U. Nackenhorst, eds. IUTAM Symposium on Computational Methods in Contact Mechanics, Hannover, Germany. Springer Netherlands, 2006. DOI: $10.1007 / 978-1-4020-6405-0$.

70. ESRD Group. StressCheck finite element simulation program. 1989. URL: https: //esrd.com. 